Check for updates

Cite this: Chem. Soc. Rev., 2021, 50,4833

Received 2nd October 2020

DOI: $10.1039 / d 0 \operatorname{cs} 00737 d$

rsc.li/chem-soc-rev

\title{
Roadmap towards solar fuel synthesis at the water interface of liposome membranes $\dagger$
}

\author{
Andrea Pannwitz, (D) $\ddagger^{* a b}$ David M. Klein, (D) $\ddagger^{a}$ Santiago Rodriguez-Jiménez, (D) ${ }^{c}$ \\ Carla Casadevall, (D) ${ }^{c}$ Hongwei Song, (D) d Erwin Reisner, (D) *c \\ Leif Hammarström (D)*d and Sylvestre Bonnet (D) *a
}

\begin{abstract}
Artificial photosynthesis has experienced rapid developments aimed at producing photocatalytic systems for the synthesis of chemical energy carriers. Conceptual advances of solar fuel systems have been inspired by improved understanding of natural photosynthesis and its key operational principles: (a) light harvesting, (b) charge separation, (c) directional proton and electron transport between reaction centres and across membranes, (d) water oxidation and (e) proton or $\mathrm{CO}_{2}$ reduction catalysis. Recently, there has been a surge of bio-inspired photosynthetic assemblies that use liposomes as nanocompartments to confine reaction spaces and enable vectorial charge transport across membranes. This approach, already investigated in the 1980s, offers in principle a promising platform for solar fuel synthesis. However, the fundamental principles governing the supramolecular assemblies of lipids and photoactive surfactant-like molecules in membranes, are intricate, and mastering membrane-supported photochemistry requires thorough understanding of the science behind liposomes. In this review, we provide an overview of approaches and considerations to construct a (semi)artificial liposome for solar fuel production. Key features to consider for the use of liposomes in solar fuel synthesis are highlighted, including the understanding of the orientation and binding of different components along the membrane, the controlled electron transport between the reaction centres, and the generation of proton gradients as driving force. Together with a list of experimental techniques for the characterisation of photoactive liposomes, this article provides the reader with a roadmap towards photocatalytic fuel production at the interface of lipid membranes and aqueous media.
\end{abstract}

\section{Key learning points}

(1) Photocatalysis on lipid membranes allows to mimic and understand natural photosynthesis and the role of membranes in it.

(2) The physical-chemical properties of the membrane, as well as the strategy used to anchor catalysts and photosensitizers to it, both influence the catalytic outcomes of membrane-embedded photocatalytic systems.

(3) Membranes are thick with respect to electron and proton transfer, and transferring them through membranes requires molecular charge carriers or transmembrane assemblies with appropriate hydro/lipophilicity.

(4) When working with dissymmetric photoactive liposomes, ion leakage and osmotic stress on the membrane should be controlled.

(5) Two-dimensional diffusion of membrane-embedded species is inherently different from 3D diffusion in bulk aqueous phases, which influences the kinetics of energy and electron transfer within lipid membranes.

\footnotetext{
${ }^{a}$ Leiden Institute of Chemistry, Leiden University, Einsteinweg 55, Leiden, 2333 CC, The Netherlands.E-mail: andrea.pannwitz@uni-ulm.de, bonnet@chem.leidenuniv.nl

${ }^{b}$ Institute of Inorganic Chemistry I, Ulm University, Albert-Einstein-Allee 11, 89081 Ulm, Germany

${ }^{c}$ Yusuf Hamied Department of Chemistry, University of Cambridge, Lensfield Road, Cambridge CB2 1EW, UK. E-mail: er376@cam.ac.uk

${ }^{d}$ Department of Chemistry - Angstrom Laboratory, Uppsala University, Box 523,

75120 Uppsala, Sweden. E-mail: leif.hammarstrom@kemi.uu.se

$\dagger$ Electronic supplementary information (ESI) available. See DOI: 10.1039/ d0cs00737d

\$ These authors contributed equally to this work.
}

\section{Introduction to photocatalysis on lipid bilayers}

\subsection{Motivation}

Natural photosynthesis provides a biological blueprint for a scalable process to store solar energy in the chemical bonds of complex organic molecules. In the light-dependent reactions of oxygenic photosynthesis, photon absorption in the thylakoid membrane drives water oxidation (eqn (1)), which provides electrons and protons for the generation of the biological 
reductant dihydronicotinamide adenine dinucleotide (phosphate) $(\mathrm{NAD}(\mathrm{P}) \mathrm{H})$ together with energy carrier molecules of adenosine triphosphate (ATP). The latter are subsequently used in the dark reaction of the Calvin cycle to fix $\mathrm{CO}_{2}$ as carbohydrates (eqn (2)), or to reduce protons (eqn (3)) in photobiological $\mathrm{H}_{2}$ production by redirecting reduced $\mathrm{NAD}(\mathrm{P}) \mathrm{H}$ towards hydrogenases in some unicellular algae and cyanobacteria under special conditions. ${ }^{1,2}$

Water oxidation half reaction $E^{\circ \prime}(\mathrm{V}) v s$. SHE at $\mathrm{pH} 7$

$$
2 \mathrm{H}_{2} \mathrm{O} \rightarrow \mathrm{O}_{2}+4 \mathrm{H}^{+}+4 \mathrm{e}^{-} 0.81
$$

Proton and selected $\mathrm{CO}_{2}$ reduction half reactions $E^{\circ \prime}(\mathrm{V}) v s$. SHE at $\mathrm{pH} 7$

$$
\begin{gathered}
6 \mathrm{CO}_{2}+24 \mathrm{H}^{+}+24 \mathrm{e}^{-} \rightarrow \mathrm{C}_{6} \mathrm{H}_{12} \mathrm{O}_{6}+6 \mathrm{H}_{2} \mathrm{O}-0.43 \\
2 \mathrm{H}^{+}+2 \mathrm{e}^{-} \rightarrow \mathrm{H}_{2}-0.41 \\
\mathrm{CO}_{2}+2 \mathrm{H}^{+}+2 \mathrm{e}^{-} \rightarrow \mathrm{CO}+\mathrm{H}_{2} \mathrm{O}-0.53 \\
\mathrm{CO}_{2}+\mathrm{H}^{+}+2 \mathrm{e}^{-} \rightarrow \mathrm{HCO}_{2}^{-}-0.39 \\
\mathrm{CO}_{2}+6 \mathrm{H}^{+}+6 \mathrm{e}^{-} \rightarrow \mathrm{CH}_{3} \mathrm{OH}+\mathrm{H}_{2} \mathrm{O}-0.38
\end{gathered}
$$

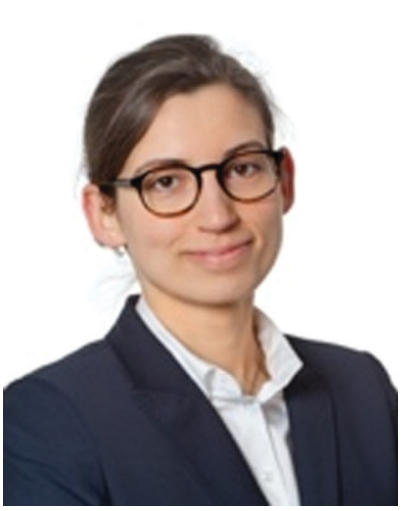

Andrea Pannwitz the SNSF. In 2020 she started her independent career as assistant professor for Inorganic Chemistry and Energy Conversion at Ulm University with a major interest in photochemical conversions at membrane interfaces.

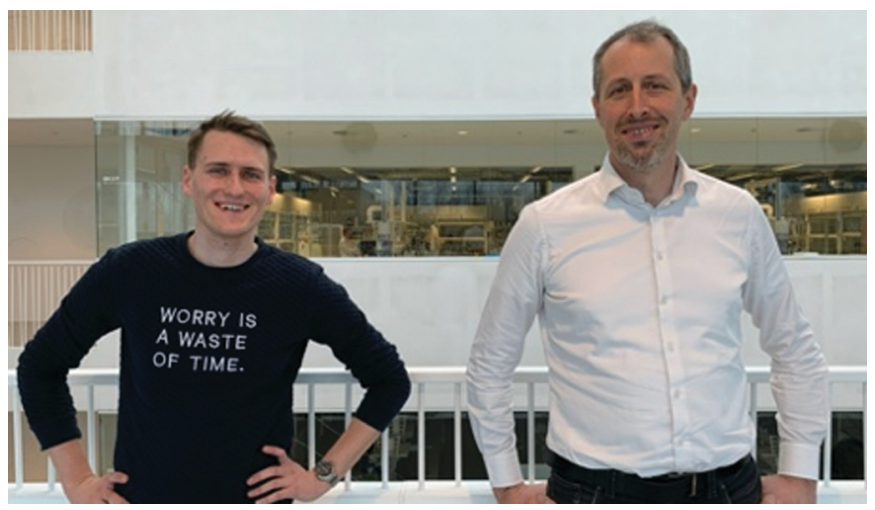

David M. Klein (left) and Sylvestre Bonnet (right)
The thylakoid membrane consists of the supramolecular assembly of lipids and additives into a flexible, fluid twodimensional membrane that hosts an array of protein units that organise the light reactions and separate them from the dark reactions. The absorption of solar energy occurs mainly via chlorophylls and accessory pigments in the light harvesting complexes (LHCs), which act as antenna for light collection (Fig. 1, left). The chlorophylls pass energy through a series of chromophores on to the reaction centres of Photosystems II (PSII) and I (PSI), where the central chlorophyll pigments P680 and P700, respectively, induce charge separation and subsequent unidirectional electron transfer. Such electron transfer occurs over a long distance, and follows an electron transport chain of small-distance electron transfer steps within the protein scaffold and redox mediators of the thylakoid membrane. ${ }^{3}$ Overall, longlived charge separation across the thylakoid membrane is achieved with high quantum efficiency via a Z-scheme energy-storage architecture. Electrons are first transferred from the photoexcited P680 in PSII to the plastoquinones (PQs) embedded in the thylakoid membrane, then to the Cytochrome $b_{6} f$ complex (Cyt $\left.{ }_{b 6 f}\right)$, where plastocyanin collects the electrons to reduce $\mathrm{P} 700^{+}$in PSI. ${ }^{3}$ The latter is an oxidised species generated from photoexcitation of PSI and electron transfer to ferredoxin (Fd, an iron-sulfur protein) and ferredoxin $\mathrm{NAD}(\mathrm{P})^{+}$reductase (FNR) in the stroma of the chloroplasts. Ultimately, P680 is regenerated in PSII by electrons liberated from water oxidation in the lumen. The unidirectional flow of electrons and protons across the membrane generate a proton gradient that powers the conversion of ADP to ATP by ATP synthase $\left(\mathrm{ATP}_{\text {ase }}\right.$, Fig. 1, left).

A crucial feature of the thylakoid membrane is that it separates the oxidative from the reductive catalytic centres by compartmentalisation of the different redox half-reactions within the chloroplast lumen and stroma (Fig. 1, left). Such organisation minimises charge recombination and chemical back reactions, which by recombining holes and electrons, or oxidised and reduced molecular species, are detrimental for the reaction. PSI and $\mathrm{ATP}_{\text {ase }}$ do not fit into the space between the stacked membranes and are therefore mainly located in the stromal lamellae. PSII is located in the grana lamellae and thereby spatially separated from PSI and $\mathrm{ATP}_{\text {ase }}$, preventing the

David M. Klein received his BSc's degree in Molecular Science \& Technology from the University of Leiden and Delft in 2015 and his MSc's degree in Chemistry in 2017 from the University of Leiden. He is currently working as a PhD in the laboratory of Sylvestre Bonnet at Leiden University supported by an HRSMC fellowship. His work focusses on the design of liposomes functionalised for artificial photosynthetic purposes, such as transmembrane electron transfer and photocatalytic water splitting.

Sylvestre Bonnet is Professor for bioinorganic chemistry at Leiden University. His research focuses on photoactive metal complexes in biological and biomimetic environments, with particular interest in photocatalysis at lipid bilayers, upconversion, and light-activated metallodrugs. 
uncontrolled spill-over of excitons from PSII to PSI. ${ }^{4}$ This heterogeneity of the thylakoid membrane maximises the packing density of photosynthetic complexes and plays an important role in optimising light harvesting and energy transfer under changing light conditions. In contrast, established approaches in artificial photosynthesis rarely take compartmentalisation into account. ${ }^{5-7}$

Mimicry of natural photosynthesis by synthetic design is an appealing strategy for the development of sustainable methodologies to produce solar fuels. Apart from the electrocatalytic oxidation of water into oxygen with the concomitant release of protons, and the reduction of $\mathrm{CO}_{2}$ (eqn (4-6)) and water (eqn (3)) into carbon-based fuels and $\mathrm{H}_{2}$, respectively, light harvesting with various photosensitisers and charge separation have been investigated. The concept of using self-assemblies in biomimetic lipid bilayer membranes for artificial photosynthesis was already approached in the 70's and 80's, ${ }^{7}$ but is receiving increased attention today due to the dramatically improved choice of catalysts and photosensitisers that can be spatially arranged to generate transmembrane potentials and compartmentalised artificial photosynthetic systems (Fig. 1, right). ${ }^{7,8}$ The use of the spherical membranes of liposomes as biologic mimics of the thylakoid membrane is a promising approach to confine redox half reactions, facilitate charge separation, and avoid crossreactivity. While synthetic catalysts are continuously improving in terms of stability and activity in solar-to-chemical conversion, nature also provides evolutionarily optimised biological catalysts (enzymes) that are also appealing for building semiartificial photosynthetic systems due to their excellent catalytic activity. ${ }^{9}$ Amphiphilic polymers have also been proposed as alternative for lipids, as they can be tuned to self-assemble into thicker and often more solid membranes, though sometimes at the cost of

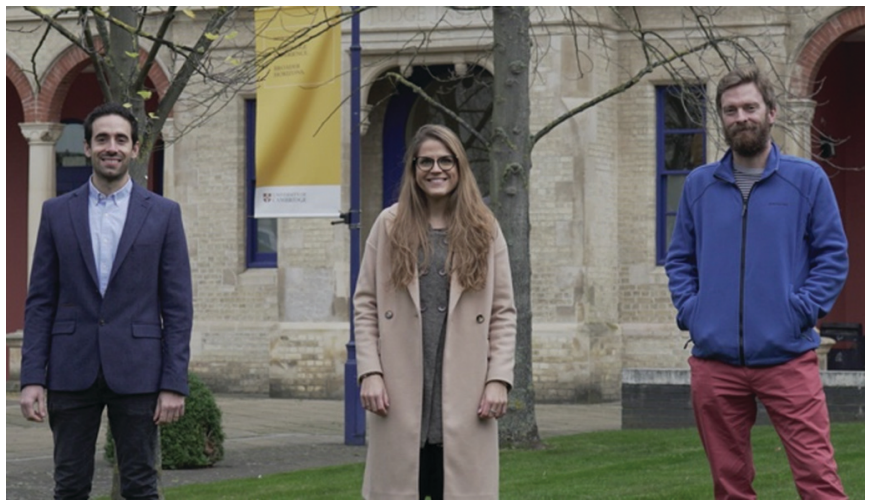

From left to right: Santiago Rodríguez-Jiménez, Carla Casadevall and Erwin Reisner

Santiago Rodriguez-Jiménez obtained his PhD in chemistry at the University of Otago under the supervision of Prof. Sally Brooker, where he worked on the design and synthesis of spin-switching iron complexes for chemo-sensing applications. After his PhD, he worked in the same group as a postdoctoral research fellow focusing on the synthesis and electrode immobilisation of molecular hydrogen evolution electrocatalysts. Subsequently, he joined the group of Erwin Reisner as a postdoctoral researcher working on artificial photosynthetic self-assembled molecular systems.

Carla Casadevall obtained her PhD degree in chemistry in 2019 at the Institute of Chemical Research of Catalonia (ICIQ) under the guidance of Prof. Julio Lloret-Fillol. Her PhD sought a fundamental understanding of the mechanisms involved in artificial photosynthesis, as well as the development of new sustainable methodologies to produce solar fuels and fine chemicals. Then, she joined the group of Erwin Reisner as a BBSRC postdoctoral researcher and now she has started in the same group as a Marie Curie fellow, working on hybrid-materials for solar fuel production.

Erwin Reisner is Professor of Energy and Sustainability at the University of Cambridge and his research focusses on the development of solar-driven processes for the sustainable synthesis of fuels and chemicals.

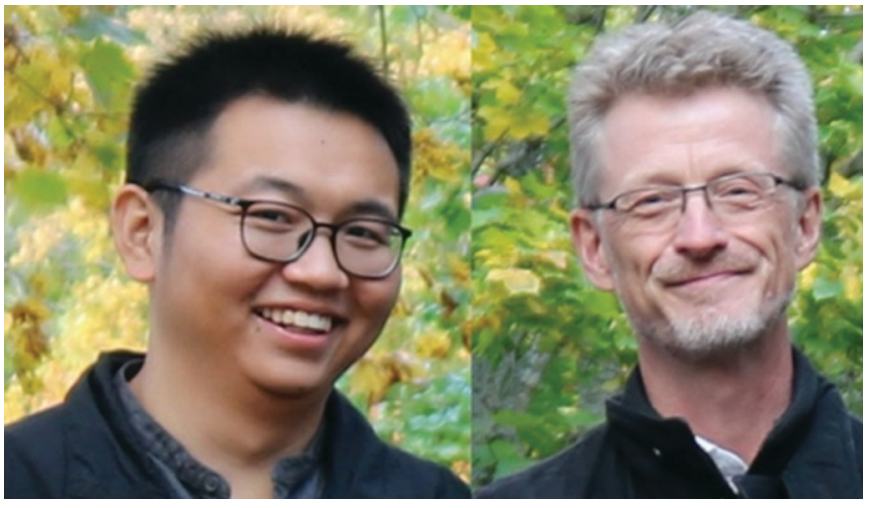

Hongwei Song (left) and Leif Hammarström (right)

Hongwei Song obtained his PhD degree in Physical Chemistry in 2019 at Institute of Chemistry, Chinese Academy of Sciences (ICCAS) under the supervision of Prof. Andong Xia, where his work focused on the understanding of ultrafast elementary photochemical processes of organic molecules in liquid solution, as well as the development of new optical spectroscopic techniques. Then, he joined the group of Prof. Leif Hammarström as a postdoctoral researcher, working on mechanisms of artificial photosynthesis. Leif Hammarström obtained his PhD degree in Physical Chemistry at Uppsala University in 1995, for his thesis "Electron Transfer in Lipid Vesicles". After postdoctoral work on inorganic photochemistry at University of Bologna, he began his independent career in Uppsala, where he since 2004 is Full Professor. His main research activities concern mechanisms of artificial photosynthesis: photoinduced electron transfer, proton-coupled electron transfer, mechanisms of solar fuel catalysts and dye-semiconductor systems. He is Chairperson of the Swedish Consortium for Artificial Photosynthesis. 

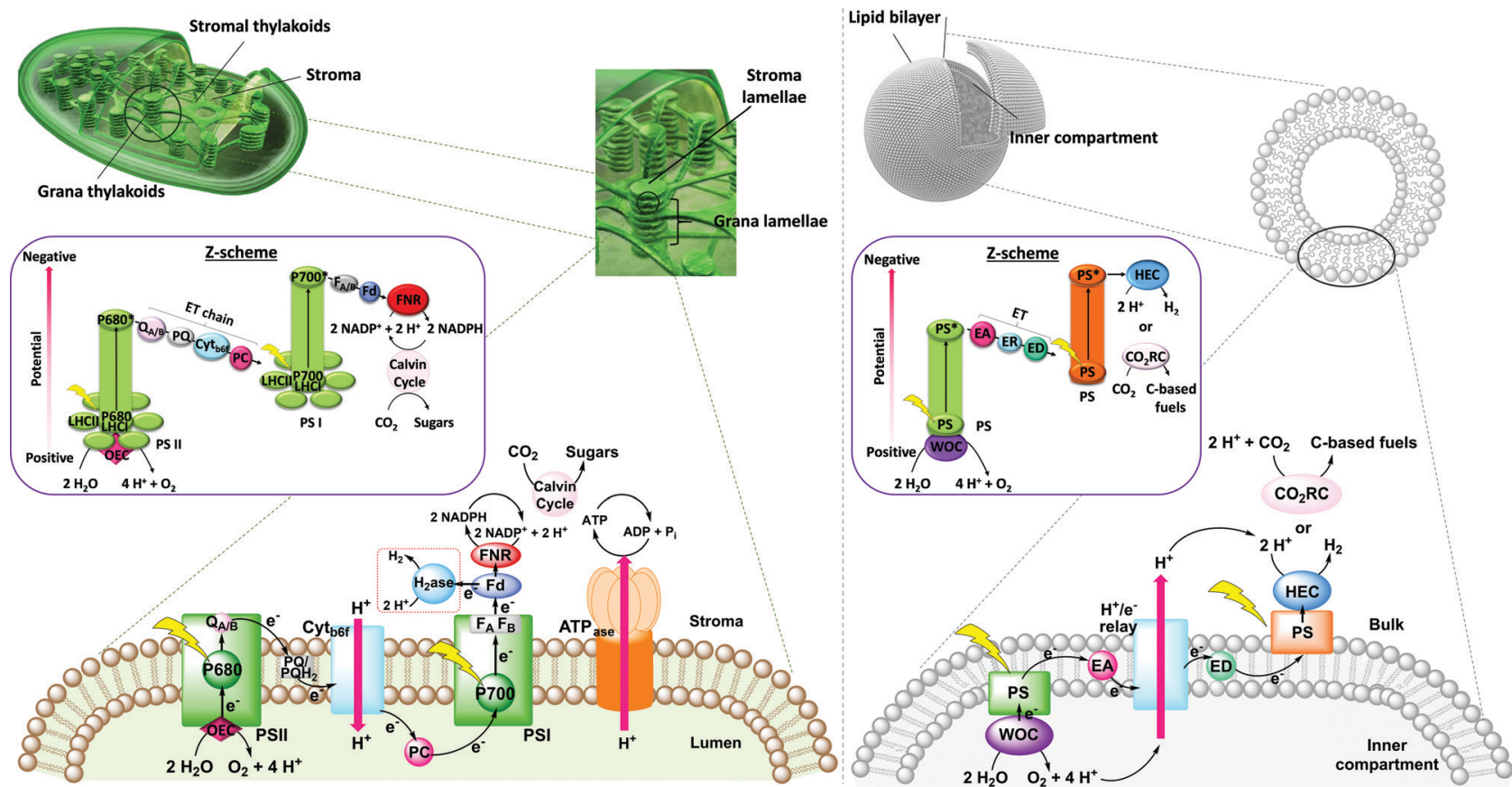

Fig. 1 Left: Scheme of a natural chloroplast showing light-induced charge transfer across the thylakoid membrane, connecting PSII, PSI and the reaction centres for $\mathrm{CO}_{2}$ reduction and ATP generation. The left inset shows the respective energy-storing Z-scheme pathway. Right: Scheme of a synthetic vesicle as a platform for an artificial photosynthetic assembly. The right inset shows the respective Z-scheme. PSI = photosystem I, PSII = photosystem II, Ph = pheophytin, $\mathrm{PQ}=$ plastoquinone, $\mathrm{PC}=$ plastocyanin, $\mathrm{b}_{6} f=$ cytochrome $\mathrm{b}_{6} \mathrm{f}, \mathrm{Fd}=$ ferredoxin, $\mathrm{FNR}=$ ferredoxin- $\mathrm{NADP} \mathrm{P}^{+}$reductase, $\mathrm{LHCl}=$ light-harvesting complex I, LHCII = light-harvesting complexes II, OEC = oxygen evolving complex, P680 and P700 = reaction centres in PSII and PSI respectively, WOC = water oxidation catalyst, $\mathrm{PS}=$ photosensitiser, $\mathrm{EA}=$ electron acceptor, $\mathrm{ED}=$ electron donor, $\mathrm{HEC}=$ hydrogen evolution $\mathrm{Catalyst} \mathrm{CRC}=\mathrm{CO}_{2}$ reduction catalyst, and $E R=$ electron relay.

biocompatibility (e.g., to accommodate transmembrane proteins). Photocatalytic polymersomes are also part of a larger effort to develop compartmentalised vesicle-based catalytic systems ${ }^{10-13}$ for tandem catalysis, which has been reviewed elsewhere and remains out of the scope of this article. This roadmap focuses on lipid-based photocatalytic vesicles for biomimetic solar fuel production, provides guidelines on how to construct such liposomes, and helps understanding the basic physicalchemical concepts, opportunities, and challenges, of using self-assembled lipid bilayer membranes as a supramolecular platform for (semi)artificial photosynthesis.

\subsection{Lipid bilayers}

Lipid bilayers are supramolecular self-assemblies formed from amphipathic lipids in an aqueous solution. Generally speaking, amphipathic (or amphipolar) molecules consist of a polar moiety (i.e., a hydrophilic head group), covalently connected to a non-polar hydrophobic part (e.g., an alkyl chain). Usually, lipids are also amphiphilic in the sense that they possess affinity for both aqueous and oil-like phases, with their preference expressed by their $\log P$ value (see below). Most amphipathic molecules are also amphiphilic, and consequently both terms "amphiphilic" and "amphipathic" are usually used as synonyms, although they are conceptually different. One should also note that some lipids can be plain hydrophobic, such as cholesterol or fat (triglycerids), in which case they are not amphipathic enough to generate bilayers in water. In all cases, for amphiphilic lipid molecules the polar head groups interact with water via iondipole or dipole-dipole interactions when exposed to an aqueous environment, whereas the interaction of the hydrophobic alkyl chain is unfavourable with water, leading to the hydrophobic effect that causes the hydrophobic groups to interact. In the case of lipids, the resulting self-assembled structure is a bilayer membrane, which generates an interface between the water phase and the more hydrophobic environments of the interior of the membrane. This hydrophobic environment can also be observed in oil-water emulsions, in foams, or in micelles-containing aqueous solutions.

Due to their amphiphilicity, lipids are also surface-active and hence surfactants. Surface activity and interfacial behaviour can be measured via tensiometry (surface pressure measurements). At a low concentration in water, surfactant amphiphiles concentrate at the interface to air, and with increasing surfactant bulk concentration, the surface tension is increasingly lowered with no aggregation being observed in the bulk solution. Increasing the surfactant concentration further will reach a point known as the critical micelle concentration (CMC), where aggregates start forming in the bulk aqueous phase. At concentrations above the CMC, only a very small fraction of the surfactant is dissolved as monomers in water $\left(c_{\max }=\mathrm{CMC}\right)$, while all other molecules are dynamically forming self-assembled aggregates. Depending on the surfactant's molecular geometry, different self-assemblies 
a) Classification

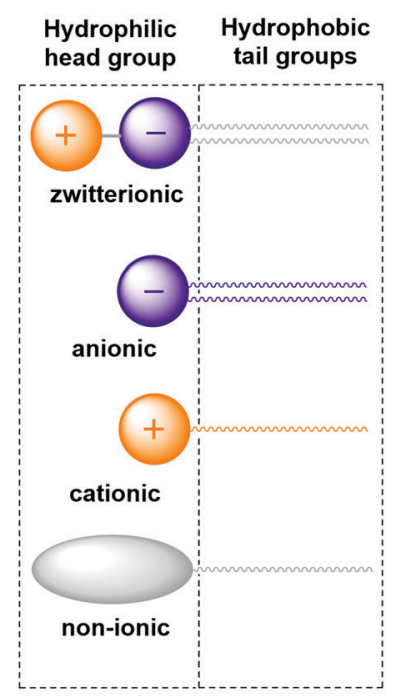

b) Examples

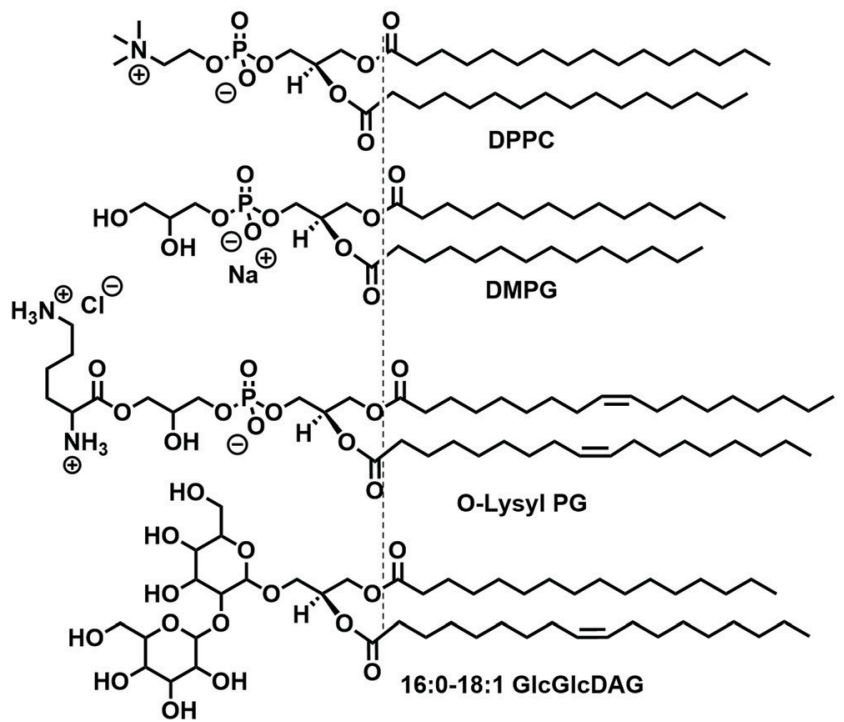

Fig. 2 (a) Classification ${ }^{14,15}$ and (b) practical examples of bilayer-forming amphiphiles: DPPC, DMPG, O-Lysyl PG, and 16:0-18:1 GlcGlcDAG.

may form, such as micelles, reverse micelles, bilayers, and vesicles. Lipids typically form bilayer membranes and vesicles. Depending on the lipids, addition of salts and differences in temperature may induce changes in aggregation dynamics and hence in the CMC.

Lipids can generally be classified according to their head groups: zwitterionic, anionic, cationic, and non-ionic. ${ }^{14}$ Examples for each class of bilayer-forming lipids are depicted in Fig. 2: the zwitterionic 1,2-dipalmitoyl-sn-glycero-3-phosphocholine (DPPC), the anionic 1,2-dimistroyl-sn-glycero-3-phosphonate(1'-rac-glycerol) (sodium salt) (DMPG), the cationic 1,2-dioleoyl-sn-glycero-3-[phospho-rac-(3-lysyl(1-glycerol))] (chloride salt) (O-Lysyl PG), and the non-ionic 1-palmitoyl-2-oleoyl-3-bis( $\beta$-D-glucosyl)-sn-glycerol (16:018:1 GlcGlcDAG). Further distinction can be made according to the counterion $\left(\mathrm{Cl}^{-}, \mathrm{Br}^{-}, \mathrm{I}^{-}, \mathrm{Na}^{+}, \mathrm{K}^{+}\right.$etc. $)$or the chemical character of the alkyl chain (e.g., hydrocarbon or fluorocarbon, saturated or unsaturated).

A common way to characterise the hydrophilicity and hydrophobicity of a molecule is to determine its water-octanol partition coefficient $(\log P)$. This dimensionless number is obtained by measuring experimentally the concentrations $c_{\text {oct }}$ and $c_{\text {water }}$ of the molecule in each phase of a biphasic $n$-octanol/water mixture at the thermodynamic equilibrium. Then, the decadic logarithm, $\log P$, is calculated as follows:

$$
\log P=\log \left(c_{\text {oct }} / c_{\text {water }}\right)
$$

Typically, hydrophilic compounds like tetraethylammonium iodide $\left(\mathrm{Et}_{4} \mathrm{~N}^{+} \mathrm{I}^{-}\right)$have a low negative $\log P$ value $(-2.82)^{16}$ and more hydrophobic compounds like triethylamine $\left(\mathrm{Et}_{3} \mathrm{~N}\right)$ have a high, positive $\log P$ value $(+1.44) .{ }^{16}$ Amphiphilic molecules have a $\log P$ value close to zero, i.e. at the equilibrium, they distribute in similar quantities in the octanol and aqueous phases. For example, nitromethane has a $\log P=0.08,{ }^{16}$ even if this molecule is not expected to have surface-active properties. On the other hand, decyltrimethylammonium iodide $(\mathrm{C} 13, \log P=-0.16)^{16}$ has one carbon less than the water-soluble tributylethylammonium iodide (C14, $\log P=-1.30),{ }^{16}$ but due to its single long alkyl tail it is more hydrophobic, also reaching a zone of less negative $\log P$ values typical for amphiphilic molecules. In addition, due to its dissymmetric "head-tail" geometry it has surface-active properties, which is also typical for lipids. One should note that other concepts to characterise surfactant-like molecules have been proposed, such as the "hydrophilic lipophilic balance" (HLB). This a) Bilayers and vesicles

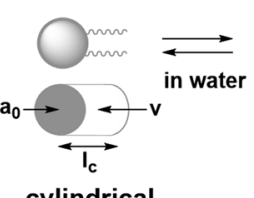

cylindrical

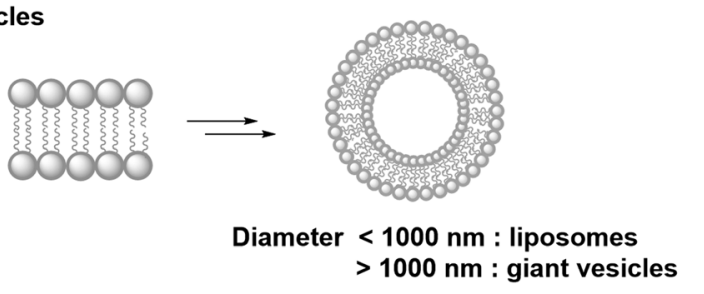

b) Micelles

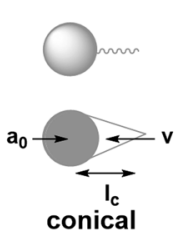

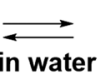

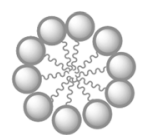

Spheres: diameter $<10 \mathrm{~nm}$

Rods: length > diameter $<10 \mathrm{~nm}$

Fig. 3 (a) Self-assembly of cylindrical amphiphiles in water into bilayers and vesicles. (b) Self-assembly of conical amphiphiles in water into spherical and rod-shaped micelles. 
numerical approximation characterises the interfacial behaviour of an amphiphile in the presence of a mixture of an organic solvent and water, as described in details elsewhere. ${ }^{14}$

In absence of organic co-solvents, amphiphiles dissolved in water usually form either membrane bilayers or micelles, depending on their geometry and steric properties. ${ }^{17}$ For bilayer formation the most suitable geometry of an amphiphile is close to a cylindrical shape (Fig. 3a). On the other hand, to obtain spherical and rod-shaped micelles a conical shape of the amphiphile is required (Fig. 3b). To assess a priori if an amphiphile forms bilayers or micelles, the packing parameter can be used:

$$
x=v /\left(a_{0} \cdot l_{\mathrm{c}}\right)
$$

where $v$ is the volume of the hydrophobic part, $a_{0}$ is the area of the headgroup at the surface, and $l_{\mathrm{c}}$ is the length of the hydrophobic tail. ${ }^{17}$ The values for $v, a_{0}$, and $l_{\mathrm{c}}$ can be obtained from crystallographic data of the amphiphile or molecular modelling. Spherical micelles are the preferred structure when $x \leq 1 / 3$, non-spherical micelles when $1 / 3<x<\frac{1}{2}$, cylindrical or rod-like micelles when $x \approx \frac{1}{2}$, above $x=\frac{1}{2}$ various interconnected structures, and finally curved to planar bilayers when $x$ approaches or equals $1 .^{17}$ For example, phospholipids such as DPPC have commonly $x>2 / 3$ and self-assemble into curved bilayers, i.e., vesicles. ${ }^{17}$ The size of these self-assembled micelles or lipid bilayer vesicles can be measured via dynamic light scattering (DLS). As discussed before, these aggregates only form at bulk lipid concentrations higher than the critical micelle concentration (CMC). Bilayer-forming lipids usually have a very low CMC compared to micelle-forming amphiphiles. For instance, the zwitterionic phospholipid DPPC has a CMC of $4.6 \times 10^{-10} \mathrm{M},{ }^{15}$ while the micelle-forming hexaethylene glycol monododecyl ether has a CMC of $8.7 \times 10^{-5} \mathrm{M} \cdot{ }^{14}$ Overall, the self-assembly of lipids depends on many intrinsic molecular properties of a lipid such as its charge, rigidity, size, and the hydrophilicity/hydrophobicity of its polar and apolar parts; but it also largely depends on the experimental conditions, including (and not limited to) the lipid bulk concentration, the temperature, the presence of salts or additives in the aqueous solution, and the composition of a lipid mixture, for example.

\subsection{Individual components for artificial photosynthesis at lipid bilayers}

Several synthetic or biological functional components are necessary to build a compartmentalised system for artificial photosynthesis. ${ }^{6}$ Basically, these functional molecules can be divided into four main categories: photosensitisers, catalysts, sacrificial reagents, and charge carriers. Important considerations have been established over the years to target these components towards different regions of the lipid bilayer and construct photocatalytic membranes. These consideration can be sorted into the four following general strategies (Fig. 4): (a) covalent attachment of the functional group to a lipid that inserts in the membrane, (b) electrostatic attraction of charged functional molecules to the charged head groups of the lipids, (c) integration of the functional group into an amphiphilic a)

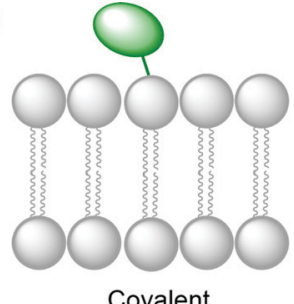

c)

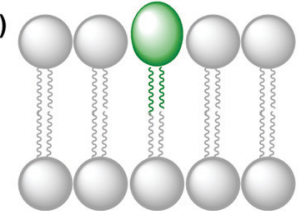

Amphiphilic - at interface b)

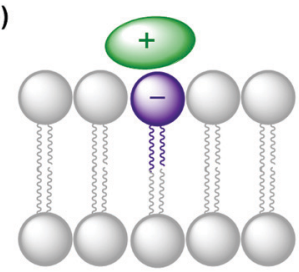

Electrostatic

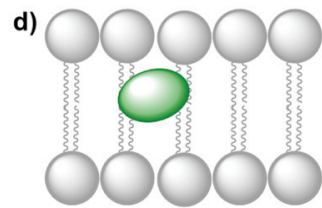

Hydrophobic - in membrane core

Fig. 4 Strategies to integrate functional molecules in lipid bilayer membranes.

molecule, which targets the interface between the bulk water phase and the hydrophobic core of the lipid membrane, and (d) building enough hydrophobicity in the functional molecule to embed it within the hydrophobic core of the membrane.

Photosensitisers. A photocatalytic cycle starts with irradiation of a photosensitiser (PS), yielding an electronically excited species, PS $^{*}$, which can act as a strong oxidant or reductant (Fig. 5). ${ }^{18}$ Quenching of the excited state via electron transfer with an electron donor (ED, reductive quenching), or electron acceptor (EA, oxidative quenching) generates the respective reduced $\left(\mathrm{PS}^{-}\right)$or oxidised $\left(\mathrm{PS}^{+}\right)$species. These reactive species may transfer their charge to the catalyst which undergoes further substrate reduction or oxidation, respectively. However, at each step of this charge transfer chain charge recombination may occur between an oxidised donor and a reduced acceptor. Achieving efficient artificial photosynthesis requires productive

\section{Photocatalytic cycle}

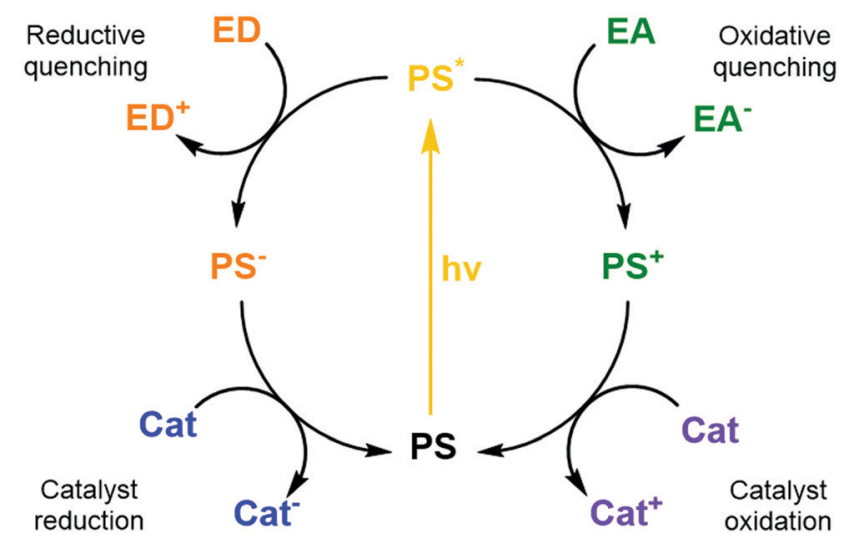

Fig. 5 Schematic photocatalytic cycles of a PS including quenching reactions with an ED or EA and the reductive and oxidative reactions with a catalyst 
a) Photosensitisers

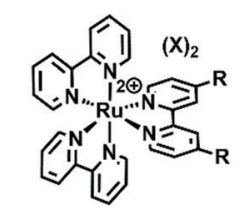

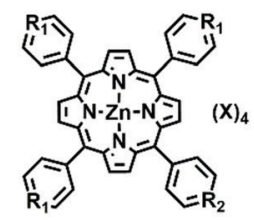

PS1 R $=\mathrm{H} ; X=\mathrm{Cl}^{-}$
PS2 R $=\mathrm{CONHC}_{12} \mathrm{H}_{25} ; X=\mathrm{PF}_{6}$
PS3 $\mathrm{R}=\mathrm{C}_{13} \mathrm{H}_{27} ; \mathrm{X}=\mathrm{PF}_{6}^{-}$

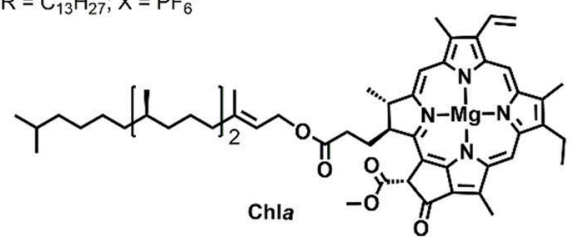

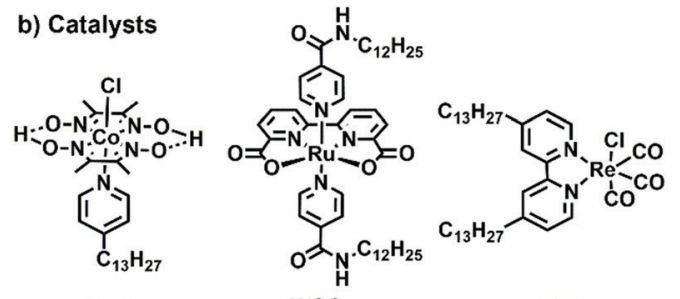

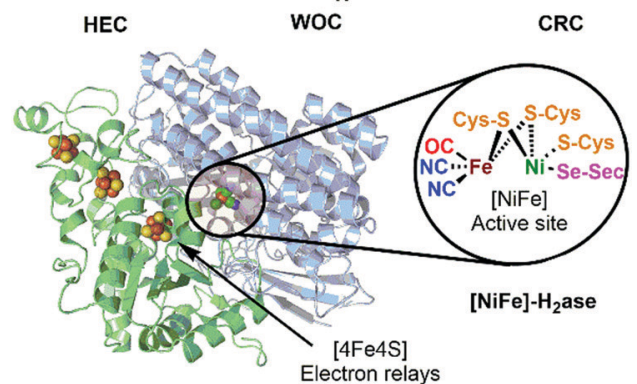

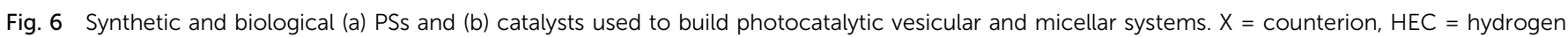

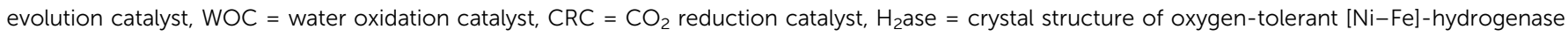
enzyme, obtained from bacterium Desulfomicrobium baculatum (pdb: 1CC1).

charge transfer towards the photoproduct to be faster than charge recombination. Furthermore, there are three additional obstacles when PSs are incorporated into lipid bilayer membranes, and all of them are related to their high local concentration. Firstly, excited states PS* may quench each other by mechanisms such as triplet-triplet annihilation between two molecules. Secondly, self-quenching (concentration quenching) may occur by other PS molecules in their ground state. Thirdly, liposome dimensions are similar to the wavelength of visible light, and at high local concentration of PS the light available to each PS is reduced. While this does not decrease harvesting efficiency of the incoming light, it means that each PS is running less efficiently, and that one maybe uses an unnecessary high amount of PS. Similarly, a high bulk concentration of PS may reduce light penetration into the solution, and make a large part of the solution rather inactive. These effects could be countered by using a low PS loading to maximise the photon energy available to the system (i.e., increase quantum yield and efficiency); ${ }^{19}$ although this strategy may also lead to a decreased solar energy conversion yield. The same considerations are important in the design of photobioreactors with photosynthetic microorganisms, and genetic reduction of the photosynthetic antenna is a strategy to find an optimum between a high cell concentration and good light penetration. The use of compartmentalisation in liposomes should be an appropriate strategy to improve the PS efficiency and performance. For instance, natural light-harvesting antennas employ close proximity of many pigment molecules and self-quenching via energy transfer is avoided by careful control of intermolecular distances and electronic coupling. ${ }^{20}$

Thermodynamics (i.e., redox potentials in ground and excited states), and kinetics (i.e., excited state lifetime and charge transfer dynamics) must be well orchestrated in different PSs to couple efficient light collection with electron transfer and catalysis within the assembled components. ${ }^{6}$ Furthermore, the spectral properties of the PS influence its ability to absorb photons. The most relevant properties here are how the absorption spectrum (extinction coefficient) of the PS overlaps with the irradiance spectrum of the light source (e.g., the sun); what are the relative quantum yields for non-radiative deactivation, electron transfer, and photon emission; and last but not least, what is the photostability of the PS under photocatalytic conditions. Ideally, the other components of the system should show little absorption where the PS absorbs most, to avoid competition for light (filter effect) and photon loss.

One of the most widely used type of PS molecules are polypyridine ruthenium(II) complexes such as $\left[\mathrm{Ru}(\mathrm{bpy})_{3}\right] \mathrm{Cl}_{2}$ (PS1, bpy $=2,2^{\prime}$-bipyridine, Fig. 6a). ${ }^{6,18}$ The positive charge of this complex and the limited hydrophobicity of the bipyridine ligands makes it quite hydrophilic $(\log P=-2.50),{ }^{21}$ which prevents it from interacting strongly with positively charged or neutral lipid bilayers. A negatively charged membrane is therefore required to attract PS1 electrostatically (Fig. 4b). An alternative strategy consists in functionalising the bpy ligand in PS1 with hydrophobic groups such as alkyl chains, to yield the amphiphilic analogues PS2 and PS3 (Fig. 6a). These molecules can be readily immobilised within lipid bilayers (Fig. 4c). For instance, PS2 was employed in lipid vesicles for photocatalytic hydrogen evolution ${ }^{19}$ and water oxidation reactions, ${ }^{22,23}$ whereas PS3 was used in unilamellar vesicles for photocatalytic carbon dioxide reduction. ${ }^{24}$ In the presence of a lipid bilayer these amphiphilic molecules may either assemble at the interface (Fig. 4c), if the alkyl chains in PS3 are not too long and the counter anion $\left(\right.$ e.g. $\left.\mathrm{Cl}^{-}\right)$solubilises the dicationic head in the water phase; or, they may integrate deeper within the membrane (Fig. 4d) with longer, more hydrophobic alkyl chains and more hydrophobic counter anions (e.g., $\mathrm{PF}_{6}{ }^{-}$). Another classical family of PSs consists in metal porphyrins and includes for example PS4, PS5 and Chla (Fig. 6a). PS4 and PS5 were immobilised in charged 
vesicles to study charge recombination ${ }^{25}$ and transmembrane charge transport, ${ }^{26}$ respectively. The biological PS Chla (Fig. 6a) acts as a visible-light harvesting unit in PSI and PSII, and was utilised in charged micelles for photocatalytic hydrogen evolution. $^{27}$

It should be noted that the integration of the PS into the membrane has often a significant effect on its electronic and photochemical properties. Despite the currently unpredictable effects of the membrane environments on the properties of the integrated components, ligand modifications (e.g. covalent attachment of alkyl groups) can tune the photophysical and redox behaviour of the PSs as well, ${ }^{18}$ or that of other active components (see below), making the spectroscopic and electrochemical characterisation of newly synthesised components fundamental to reach an efficient self-assembled system. Last but not least, the primary input in a photocatalytic system is the rate of formation of excited states, or rate of photon absorption. This photochemical input depends not only on the PS concentration, but also on the molar absorption coefficient of the PS at the irradiation wavelength, and on light intensity. The quantum yield of product formation can be calculated by dividing the measured rate of product formation by the rate of photon absorption, and multiplying this number by the number of electrons transferred photocatalytically (e.g., two for the reduction of $\mathrm{CO}_{2}$ into $\mathrm{CO}$, or four for water oxidation into $\mathrm{O}_{2}$ ).

Catalysts for solar fuel production are commonly redox active metal complexes that can accumulate charges (either electrons and/or protons) to facilitate bond-making and bondbreaking reactions such as hydrogen evolution, $\mathrm{CO}_{2}$ reduction, or water oxidation. Examples for catalysts that have been supported on lipid bilayers and micelles are depicted in Fig. 6b. Hydrogen evolution was performed with an alkylated cobaloxime-based hydrogen evolution catalyst (HEC) in zwitterionic vesicles and with a hydrogenase in charged micelles. ${ }^{19,28}$ An alkylated rhenium $\mathrm{CO}_{2}$ reduction catalyst (CRC) was employed to study photocatalytic $\mathrm{CO}_{2}$ reduction in zwitterionic vesicles. ${ }^{24}$ Photocatalytic water oxidation in vesicles was achieved with an alkylated version of a Ru-based water oxidation catalyst (WOC). ${ }^{23}$ More examples of catalysts, which could be potentially employed upon ligand modification in vesicles, are reported elsewhere. ${ }^{6,29}$

To select a suitable catalyst, several performance metrics such as catalytic activity, product selectivity, and stability, need to be considered. The catalytic activity is characterised by the turnover number $\left(\mathrm{TON}_{\mathrm{Cat}}\right)$ and turnover frequency $\left(\mathrm{TOF}_{\mathrm{Cat}}\right)$. TOF $\mathrm{Tat}_{\mathrm{Ca}}$ is a measure of the product generation rate, given as the number of moles of products produced per mole of catalyst in the system and per unit time. The $\mathrm{TON}_{\text {Cat }}$ at a certain time $t$, is the total number of product molecules generated at that moment per molecule of catalyst initially introduced in the system. While in catalysis under dark conditions, catalyst decomposition often explains why the reaction stops, in photocatalysis the end of a photocatalytic reaction may also be due to PS degradation. For photocatalytic systems it is thus equally important to assess the PS stability. Here, we refer to $\mathrm{TON}_{\mathrm{PS}}$ and $\mathrm{TOF}_{\mathrm{PS}}$ when referencing the TON or TOF of the system to the amount of PS initially introduced. It should also be noted that sometimes, $\mathrm{pH}$ changes or consumption of the sacrificial reagent may also be involved in the end of the photocatalytic reaction.

A catalyst requires a certain driving force (i.e., redox potential) to generate the catalytically active species. The difference between this catalytic onset potential and the thermodynamic potential (see eqn (1)-(6)) is known as the overpotential. In an ideal scenario, a high reaction rate is reached at a small overpotential. In a photochemical system, the "applied" potential often corresponds to the redox potential of the reduced or oxidised ground state of the PS, and it should match the overpotential requirement of the catalyst. Particularly important in $\mathrm{CO}_{2}$ reduction is product selectivity, because co-generation of $\mathrm{H}_{2}$ and other carbon-based reduction products readily occurs in $\mathrm{CO}_{2}$-saturated aqueous solutions. Stability is especially challenging in water oxidation catalysis, where organic ligands often degrade under the highly oxidising conditions and evolved $\mathrm{O}_{2} \cdot{ }^{30}$ The performance of catalysts is commonly assessed in homogeneous solution, but the hydrophobic anchoring groups and the membrane environment will affect the catalytic properties. ${ }^{29}$

Biological catalysts (enzymes) operate commonly with intrinsically low overpotentials and are therefore efficient model catalysts in semiartificial photocatalytic systems, ${ }^{2,10}$ including semiartificial photocatalytic micellar systems and electrocatalytic bilayer lipid membranes. ${ }^{29,31}$ These excellent catalytic capabilities are mainly due to their evolutionary-optimised architecture in which the protein environment facilitates the catalytic transformation at the active site by controlling substrate access and orientation as well as stabilising transition states of catalytic intermediates. Some examples are hydrogenase $\left(\mathrm{H}^{+} \leftrightarrow \mathrm{H}_{2}\right.$; Fig. 6b), formate dehydrogenase $\left(\mathrm{CO}_{2} \leftrightarrow\right.$ formate), carbon monoxide dehydrogenase $\left(\mathrm{CO}_{2} \leftrightarrow \mathrm{CO}\right)$ enzymes, and PSII for light-driven water oxidation. ${ }^{1,2,9}$ Despite their excellent performance, these enzymes are costly to isolate, fragile, and often display $\mathrm{O}_{2}$-sensitivity, which can make them difficult to handle. Their suitability for the integration in photocatalytic systems also requires consideration of cofactor dependence (e.g. NADH) and substrate affinity. The latter is expressed by the MichaelisMenten constant $\left(K_{\mathbf{M}}\right)$, which is low for high substrate affinity. The use of compartmentalised vesicles allows the encapsulation of enzymes within the vesicles with low substrate affinity and provide them with the required high local concentration of substrate as in living cells. The reader is referred to references $^{1,9}$ for a more detailed discussion on selection criteria for enzymes.

Sacrificial ED and EA reagents are widely used in artificial photosynthesis when studying half-reactions, although the ultimate goal is to use a sustainable source of charge to drive a closed redox cycle. ${ }^{32}$ They serve as reductive and oxidative equivalents used to quench a photoexcited PS (PS*, Fig. 5), or regenerate the PS after reductive or oxidative quenching by a primary quencher. This reduces the possibility for charge recombination as they commonly decompose quickly and irreversibly. The use of sacrificial reagents allows investigating and optimising individual half-reactions such as proton $/ \mathrm{CO}_{2}$ reduction or water oxidation without the demanding requirement to couple both half reactions with a single charge carrier. 
The choice of ED and EA is generally based on their redox potentials, rapid photooxidation and reduction in the presence of PS $^{*}$ and, ideally, producing harmless intermediates and products, although non-innocent decomposition by-products are sometimes generated. Some of the most commonly used ED in aqueous media for light-driven proton and $\mathrm{CO}_{2}$ reduction are mild reducing agents such as triethanolamine, ethylenediaminetetraacetic acid (EDTA), and ascorbic acid. ${ }^{32}$ On the other hand, one of the most common EA used in light-driven water oxidation reactions is the strong oxidising agent sodium peroxodisulphate $\left(\mathrm{Na}_{2} \mathrm{~S}_{2} \mathrm{O}_{8}\right){ }^{6}\left[\mathrm{Co}\left(\mathrm{NH}_{3}\right)_{5} \mathrm{Cl}\right] \mathrm{Cl}_{2}$ has been proposed as well, but its propensity to form catalytically active cobalt oxides is problematic, in particular in the context of the water oxidation reaction. One should note that the conditions used for many photocatalytic water oxidation experiments, i.e. near-neutral $\mathrm{pH}$ and $\mathrm{Na}_{2} \mathrm{~S}_{2} \mathrm{O}_{8}$ as $\mathrm{EA}$, are drastically different from the conditions in which chemically driven water oxidation catalysts are usually tested, which involves strong concentrations of cerium ammonium nitrate $(\mathrm{CAN})$ and highly acidic solution $(\mathrm{pH}=1)$.

Charge carriers are redox active species capable of transporting reducing or oxidising equivalents between different components of a photocatalytic system (Fig. 7a). A typical example is the watersoluble, dicationic methyl viologen $\left(\mathbf{M V}^{2+}\right)$, which can accept one electron to form the blue-coloured cation radical $\mathbf{M V}^{\mathbf{}}{ }^{+}$. In some cases, charge carriers can undergo proton-coupled electron transfer (PCET) and carry electrons and protons across lipid membranes; good examples are the membrane-soluble 1-methoxy- $N$-methylphenazinium cation $\left(\mathbf{M M P}^{+}\right)$, or the quinones 2,5-diphenyl-1,4benzoquinone $\left(\mathbf{Q}_{\mathbf{s}}\right)$ and decylubiquinone (dQ). ${ }^{26,33}$ The thermodynamic and kinetic features of charge carriers must also be carefully considered to ensure an efficient unidirectional electron transfer. Furthermore, the chemical stability and solubility of the charge carrier must be high in the relevant redox and protonation states (Fig. 7a). For example, in presence of $\mathrm{O}_{2}$ the one-electron reduced species $\mathbf{M V}^{\bullet+}$ is re-oxidised into $\mathbf{M V}^{2+}$, which can be problematic in full water-splitting schemes also producing $\mathrm{O}_{2}$.

The electrostatic charge of a carrier can also be exploited to enhance ET kinetics or minimise charge recombination due to electrostatic repulsion or attraction. ${ }^{25}$ Other features to consider are whether the reduced or oxidised charge charrier acts innocently, i.e. it exclusively engages in an outer-sphere ET reaction, or non-innocently, i.e. it can undergo side-reactions such as a disproportionation reaction (e.g. $2 \mathbf{M V}^{+} \rightarrow \mathbf{M V}^{2+}+\mathbf{M V}^{\mathbf{0}}$ ) or reacts with $\mathrm{O}_{2}$ present in solution. ${ }^{34}$ The affinity of the charge carrier for the catalyst should also be considered, as it could disrupt catalysis by coordinatively binding to the catalytically active a) Charge carriers

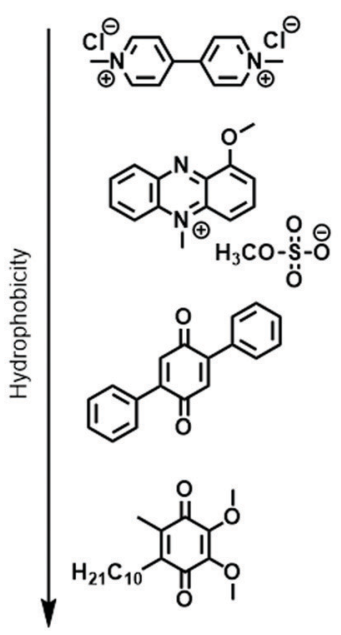

b) Biological transmembrane electron transporter

$\operatorname{MV}(\mathrm{Cl})_{2}$

$\operatorname{MMP}\left(\mathrm{SO}_{4} \mathrm{CH}_{3}\right)$

Qs

dQ

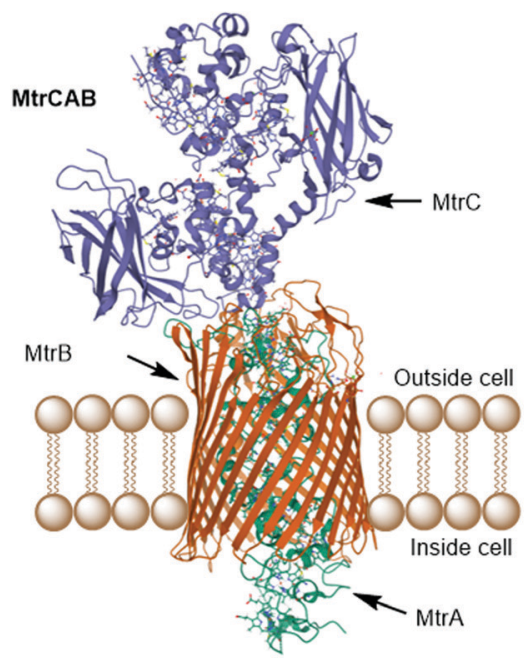

c) Artificial reaction centre

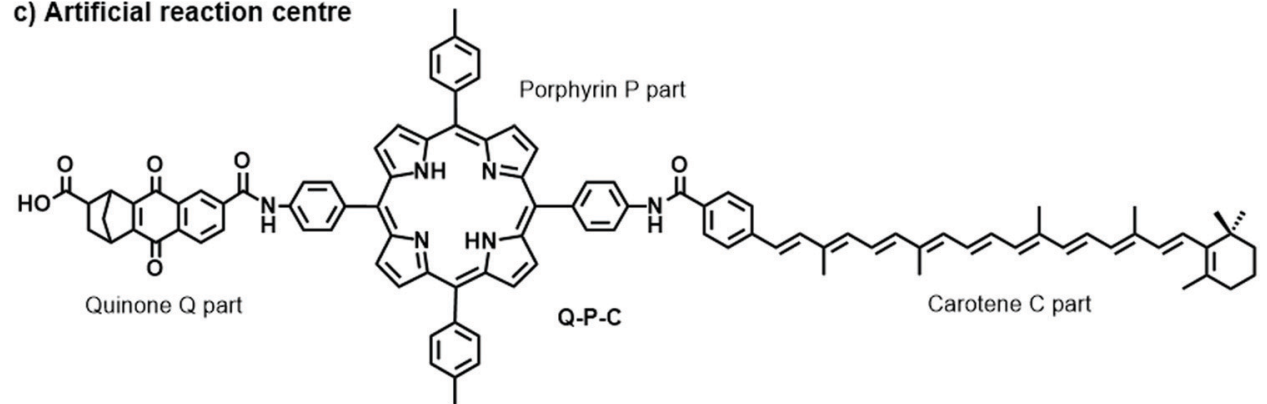

Fig. 7 Molecular structures of (a) metal-free charge carriers, (b) crystal structure of biological electron transmembrane transporter icosa-heme cytochrome protein MtrCAB from bacterium Shewanella baltica (pdb: 6R2Q), with cellular lipid bilayers and its three subunits, and (c) artificial reaction centre $Q-P-C$ used to light-induce proton transport across vesicle membranes. ${ }^{33,35}$ 
metal centre. Another aspect to consider are changes in the $\mathrm{p} K_{\mathrm{a}}$ value due to reduction or oxidation of the charge carrier, which could influence catalysis. Moreover, the effect of membrane environments can tune features of the catalyst such as its redox potentials, which are typically measured electrochemically in bulk organic solutions. In addition to the presented metal-free charge carriers (Fig. 7a), water-soluble metal-based complexes, such as cationic cobalt tris-bipyridine complex, $\left[\mathrm{Co}(\mathrm{bpy})_{3}\right]^{2+/ 3+}$, could be conceptually appropriate reductive and oxidative equivalent carriers, however because of the similarity with other synthetic components (e.g. the water oxidation or proton reduction catalyst) they may potentially act undesirably. Other hydrophilic electron relays such as $\mathrm{I}^{-} / \mathrm{IO}_{3}{ }^{-}$and $\mathrm{Fe}^{3+} / \mathrm{Fe}^{2+}$ have also been used. Compartmentalised vesicles have also been proposed that use lipophilic charge carriers to transport electrons and protons across vesicle membranes between spatially separated reaction centres. For example, membrane-bound cytochrome MtrCAB proteins (Fig. 7b) have been proposed as biological molecular wires and membrane-embedded artificial reaction centres, which artificial quinone-porphyrin-carotenoid triads (Q-P-C, Fig. 7c) $)^{33,35}$ try to mimic. Overall, transporting the reductive equivalent from the site of water oxidation to the site of proton or $\mathrm{CO}_{2}$ reduction requires charge carriers that are metastable in two different oxidation states, and do not react too quickly with the photogenerated products $\mathrm{O}_{2}, \mathrm{H}_{2}$, and/ or reduced form of carbon dioxide.

To conclude, not only the position in the membrane, but also the number of electrons exchanged by the different components of the membrane during photocatalysis, should be considered carefully. Overall, natural photosynthesis requires at least eight photons in both photosystems to extract four electrons and protons from two water molecules in PSII, and to deliver these electrons one by one for the two-electron reduction of two $\operatorname{NAD}(\mathrm{P})^{+}$molecules via Cytochrome b6f (Cyt b6f) to PSI, while protons are passed one by one through the membrane by stepwise rotation of ATPase, thereby generating ATP (Fig. 1, left). Likewise, there is often a mismatch in the number of electrons (or charges) exchanged by the different components of an artificial photosynthesis system (Fig. 1, right). Typically, one electron is exchanged between an excited photosensitizer molecule and a charge carrier such as ascorbate or $\mathrm{MV}^{2+}$, while two electrons are needed for long-distance charge transporters such as quinones or $\mathrm{MMP}^{+}$, two to eight electrons are needed for catalytic fuel production, and four electrons are provided by water oxidation. This discrepancy in principle requires careful analysis of the kinetics of each electron transfer step, to understand how they may integrate in a solar fuel production system.

\section{The hydrophobic membrane}

\subsection{Properties of the membrane}

Natural cell membranes are two-dimensional (2D) flexible liquid crystals that can adjust easily to environmental mechanic stress and provide a fluid "solvent" for membrane proteins as well as high mobility for mass transport within the membrane. Depending on

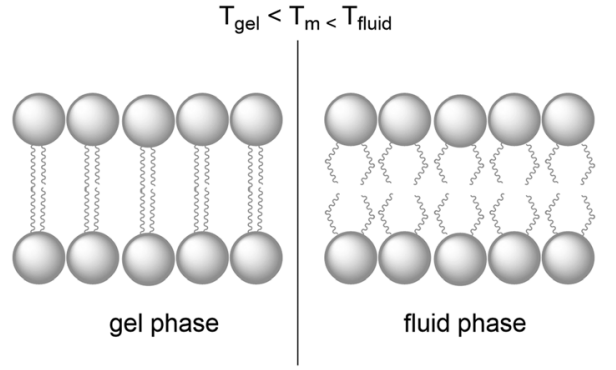

Fig. 8 A lipid bilayer changes from the rigid gel phase (left) to the mobile fluid phase (right) above the transition temperature $\left(T_{m}\right)$ of the lipid.

the application, more rigid membranes can also be prepared. ${ }^{6,24,26}$ Most membranes indeed can exist in different phases, typically a liquid-crystalline phase with high lateral diffusion of the lipids, and a gel-like or even "solid" phase, characterised by much lower lateral diffusion properties (Fig. 8). A membrane with the desired phase, flexibility, and mobility, can be constructed from either pure lipids or from lipid mixtures. In the former case, transition temperatures $\left(T_{\mathrm{m}}\right)$ from the gel phase to the liquid crystalline phase are tabulated for most lipids (see Table 1). ${ }^{15}$ Additives such as cholesterol and lipid mixtures can fine-tune the properties of the membrane. Experimentally, $T_{\mathrm{m}}$ can for example be obtained from fluorescence anisotropy measurements with a membrane-solubilised probe, ${ }^{34}$ or differential scanning calorimetry.

Molecularly, the phase of the lipid bilayer is influenced by the number and length of the alkyl chains, their degree of unsaturation, the headgroup structure (i.e. its size, electrical charge, and polarity), and the presence of additives such as other amphiphiles or cholesterol in the membrane. Saturated chains (such as DPPC) allow for closer packing than the less flexible and bulkier unsaturated chains (such as 1,2-dioleoyl-snglycero-3-phosphocholine, DOPC), and longer chains maximise hydrophobic interactions. These features explain the large variation in gel-to-liquid crystalline transition temperatures $T_{\mathrm{m}}$ between lipids such as DPPC and DOPC. The lateral and transverse diffusion of lipids and active components in the lipid bilayer are influenced by the mobility of the medium and hence the phase of the lipid bilayer. Diffusion coefficients for $2 \mathrm{D}$ lateral mobility in liquid crystalline bilayers are on the order of $\mu \mathrm{m}^{2} \mathrm{~s}^{-1}$, while the values in the gel phase are several orders of magnitude lower. The lateral mobility describes the mobility of a lipid molecule (or photoactive component in the membrane) parallel to the water-membrane interface and within one of the monolayers

Table 1 Phase transition temperature $\left(T_{m}\right)$ from liquid crystalline to gel phase of selected membrane forming lipids ${ }^{15,34}$

\begin{tabular}{lc}
\hline Lipids & $T_{\mathrm{m}}\left({ }^{\circ} \mathrm{C}\right)$ \\
\hline DSPC & 55 \\
DPPC & 41 \\
SMPC & 31 \\
DMPG & 23 \\
DOPC & -17 \\
Egg lecithin & -10
\end{tabular}


forming the membrane. Transversal diffusion from one monolayer to the other, also called "flip-flop", requires intermediates states where the hydrophilic head of the molecule is located in the hydrophobic core of the membrane; it is hence a slow process, that takes place within several hours to weeks, depending on the lipid composition and temperature. ${ }^{15}$

Unsaturated lipids in a lipid mixture usually decrease its phase transition temperature $T_{\mathrm{m}}$ and increase the lateral fluidity of a membrane, which is in principle good for electron transfer processes within the membrane. However, they also increase the photochemical membrane instability, as double bonds are prone to oxidation in the presence of light and $\mathrm{O}_{2}$, in particular with transition-metal based PSs that can quickly generate long-lived triplet excited states. In the presence of $\mathrm{O}_{2}$, these triplet excited states are indeed prone to generate singlet oxygen $\left({ }^{1} \mathrm{O}_{2}\right)$ by energy transfer, or radical oxygen-based species (such as the superoxide ion $\mathrm{O}_{2}{ }^{--}$) by electron transfer, which in turn can oxidise the unsaturated moieties in the lipid tails into peroxides, alcohols, or conjugated ketones. ${ }^{36}$ Lipid photooxidation can culminate into $\mathrm{C}=\mathrm{C}$ double bond breaking and generation of aldehydes that destabilise the membrane and make it leaky, which is counter-productive for compartmentalised photocatalysis. The introduction of unsaturated parts in photocatalytic membranes needs hence to be carefully considered, avoided if possible, and when utilised, the integrity of the membranes should be carefully verified.

The role of membrane fluidity in semi-artificial vesicular systems was investigated in a few examples. Photocatalytic hydrogen evolution in vesicles, containing membrane-bound PS2 and a homogeneous [FeFe]- $\mathbf{H}_{2}$ ase subunit mimic catalyst, performed with higher TON of $\mathrm{H}_{2}$ with low $T_{\mathrm{m}}$ lipids, such as DOPC and DMPC, than with DPPC or 1,2-distearoyl-sn-glycero-3phosphocholine (DSPC). ${ }^{37}$ On the other hand, photocatalytic water oxidation in vesicles, ${ }^{22}$ containing membrane-bound PS2 and $\left[\mathrm{Ru}(\mathrm{ddp})(4 \text {-picoline })_{3}\right]$ (ddp $=$ 4-dodecyl-2,6-dipicolinate) catalyst, showed the opposite trend, i.e. lower TON of $\mathrm{O}_{2}$ were obtained with the low $T_{\mathrm{m}}$ lipid DOPC than with lipids with higher $T_{\mathrm{m}}$ such as DMPC and 1-stearoyl-2-myristoyl-sn-glycero3-phosphocholine (SMPC). Thus, although basic understanding of the physical-chemical principles underlying photochemistry in unsaturated membrane is available, there has been no thorough investigation of the detailed role of unsaturations in lipids and of $T_{\mathrm{m}}$ on the performances of photocatalytic membranes.

\subsection{Structural integrity of liposomes}

Although liposomes are well-defined supramolecular systems with excellent kinetic stability in solution, photoreactive liposomes sometimes become unstable in the dark or during photocatalysis. For example, they may coalescence into very large assemblies by aggregation, in particular with uncharged lipids; alternatively, the membrane may rupture due to the formation of holes or due to photochemical oxidation of its lipids components; or the membrane may become destabilised by external agents, for example, a detergent, or a photoproduct. These phenomena may be prevented by the following "tricks": (a) The bilayer may be strengthened by introducing additives, e.g. 20 percent cholesterol;

(b) Electrostatic repulsion may be embedded by design, e.g. by doping the liposomes with positively or negatively charged lipids to generate charged membrane interfaces. For example, $20 \%$ charged lipids were mixed in neutral or zwitterionic lipids, ${ }^{38}$ or amphiphilic active components with charged head groups were added to the membrane. ${ }^{25}$

(c) Steric hindrance may be added by introducing polyethylene glycol-containing lipids in the lipid mixture forming the membrane. Here as well, the PEG group prevents the liposomes to aggregate. A typical example is the anionic lipid 1,2-dimyristoylsn-glycero-3-phosphoethanolamine- $N$-[methoxy(polyethylene glycol)2000] (NaDSPE-PEG2K), which is added ( $\sim 1 \mathrm{~mol} \%$ ) to for example zwitterionic lipid membranes based on e.g. 1,2-dimyristoyl-snglycero-3-phosphocholine (DMPC). ${ }^{39}$

\subsection{Osmotic pressure}

Controlling the bulk concentrations of membrane-impermeable solutes, and hence the osmotic pressure of the aqueous solutions on both sides of the liposome membrane, is a crucial consideration when preparing photocatalytic liposomes, especially where the inner aqueous compartment and outer bulk solution do not have the same composition (see Section 3.1). The osmotic pressure of a solution onto a bilayer membrane expresses the pressure which is required to stop water from diffusing through the lipid bilayer by osmosis. The two solutions on both sides of the membrane may have different or equal osmotic pressures, which will determine the electrochemical and mechanical balance of the membrane (see below). For each solution, the contribution $\pi_{i}$ (in Pa) of each solute $i$ to the total osmotic pressure $\pi$ can in principle be calculated by the following equation:

$$
\pi_{i}=j_{i} \cdot c \cdot R \cdot T
$$

where $j_{i}$ is the Van't Hoff factor of solute $i$ (dimensionless), $c$ is the concentration of the solute (in $\mathrm{mol} \mathrm{L}^{-1}$ ), $R$ is the ideal gas constant (8,314.46261815324 $\mathrm{Pa} \mathrm{K}^{-1} \mathrm{~L} \mathrm{~mol}^{-1}$ ), and the temperature $T$ (in K). The Van't Hoff factor $j_{i}$ is calculated based on its degree of dissociation $\alpha_{i}$, and the number of resulting molecular/ ionic species, $n_{i}$, obtained upon dissolution of the solute $i$ :

$$
j_{i}=1+\alpha_{i}\left(n_{i}-1\right)
$$

Eqn (10) gives, for a simple solution containing a nondissociating solute such as glucose $\left(n_{i}=1\right), \alpha_{i}=0$ and $j_{i}=1$. A fully dissociating solute has $\alpha_{i}=1$, thus $\mathrm{NaCl}\left(n_{i}=2\right)$ yields $j_{i}=2$, while $\mathrm{MgCl}_{2}\left(n_{i}=3\right)$ yields $j_{i}=3$. However, for any solute that is dissociating incompletely $\left(0<\alpha_{i}<1\right)$, such as carboxylic acids, or for the phosphates of a phosphate buffer, it may become difficult to calculate $j_{i}$ and hence $\pi_{i}$. In addition, eqn (9) and (10) account for perfect solutions only, and might deviate from reality when ions have multiple charges, or when concentrations are not low $\left(>10^{-2} \mathrm{~mol} \mathrm{~L}^{-1}\right)$. Last but not least, buffer solutions might be too complex, or have unknown components, which makes the calculation of its osmotic pressure impossible. In such cases, the total osmotic pressure of a solution can simply be measured 


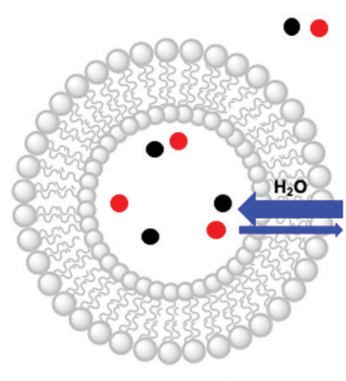

a) Hypotonic

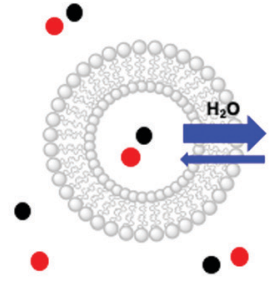

b) Hypertonic

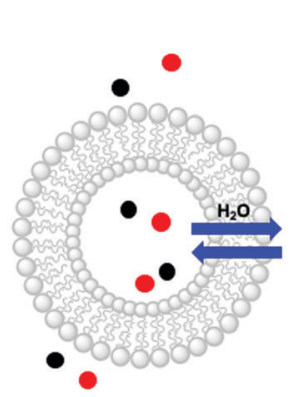

c) Isotonic

Fig. 9 Different ions and ion concentrations lead to osmotic pressure, which results in (a) a hypotonic, (b) hypertonic, or (c) isotonic situation. Amount of red and black dots represent relative concentrations.

experimentally, in osmole per litre (abbreviated as Osm $\mathrm{L}^{-1}$, a non-SI unit), using an osmometer. For example, a $0.125 \mathrm{M}$ ethylenediaminetetraacetic acid buffer at $\mathrm{pH}=8$ has an osmotic pressure of $374 \mathrm{mOsm} .{ }^{23}$ To obtain mechanically stable liposomes, the experimental osmotic pressures of the two solutions on both sides of the liposome membrane, whatever they contain, should be equal.

In a liposome, three scenarios can indeed be distinguished: a hypotonic, a hypertonic, and an isotonic situation (Fig. 9). In a hypotonic scenario, the solution on the outside of the lipid bilayer has a lower concentration of membrane-impermeable solutes. This scenario results in a net flow of water from the bulk towards the inner aqueous compartment, in turn leading to the swelling of the liposome (Fig. 9a). In extreme cases, the liposomes may burst, releasing their interior towards the outside. A hypertonic situation describes the opposite scenario, which causes a net flow of water from the inner compartment towards the bulk resulting in liposome shrinkage (Fig. 9b). For instance, liposomes based on egg yolk phosphatidylcholine with a typical hydrodynamic diameter of $140-150 \mathrm{~nm}$ as determined by DLS, decreased by $20 \mathrm{~nm}$ upon addition of $\mathrm{NaOH}$ (to $\mathrm{pH}>11$ ) to the bulk (outside) solution. ${ }^{40}$ This physical behaviour was assigned to the impermeability of the lipid bilayer towards $\mathrm{Na}^{+}$. Finally, in the isotonic situation (Fig. 9c) the total contribution to the osmotic pressure of all membrane-impermeable solutes in the inner aqueous compartment and in the bulk, are balanced, resulting in a net-zero movement of water, and a time-independent size of the liposomes. A hypothetical dilution of such a liposome sample with pure water would result in a decrease of the bulk concentration of $\mathrm{NaCl}$ and hence yield a hypotonic situation and swelling of the liposomes. To conclude, liposomes are very dynamic supramolecular systems and simple dilutions with MilliQ water for example can lead to drastic changes in the size and integrity of the liposomes. In principle, addition of redox or photoactive components on one side of a liposome, should always be done with isotonic solutions, prepared with an osmometer.

\subsection{Membrane permeability and leakage}

2.4.1 Membrane permeability: phenomenology. The formation of a lipid bilayer results in the separation of two distinct aqueous interfaces, namely that in contact with the inner aqueous compartment and the bulk solution. Importantly, molecules can diffuse across the lipid bilayer from one interface to the other, but the membrane permeability is different for each molecule and lipid composition. The diffusion rate can be defined by eqn (11) and (12), derived from Fick's first law:

$$
J_{i}=K_{i} \cdot A \cdot\left(C_{i, \text { outside }}-C_{i, \text { inside }}\right)
$$

and

$$
K_{i}=\left(P \cdot D_{i}\right) / d
$$

where $J_{j}$ is the rate of diffusion of solute $i$ (in mol s${ }^{-1}$ ), $K_{i}$ is the permeability coefficient of solute $i\left(\mathrm{~cm} \mathrm{~s}^{-1}\right), \mathrm{A}$ is the surface area of the membrane (in $\mathrm{cm}^{2}$ ), $C_{i, \text { outside }}-C_{i \text {,inside }}$ is the concentration difference of solute $i$ across the membrane (assuming $C_{i, \text { outside }}>C_{i \text {,inside, }}$ in $\mathrm{mol} \mathrm{cm} \mathrm{cm}^{-3}$ ), $P$ is the partition coefficient of the solute in the membrane that may be approximated to that used in $\log P$ measured at $n$-octanol/water biphasic mixtures, $D_{i}$ is the 2D diffusion constant of solute $i$ (in $\mathrm{cm}^{2} \mathrm{~s}^{-1}$ ), and $\mathrm{d}$ is membrane thickness (in $\mathrm{cm}$ ). As can be observed from eqn (11), a high concentration gradient and a high surface area of the membrane result in a higher rate of diffusion, whereas a thick membrane results in a lower rate of diffusion. The thickness of the membrane depends on the character of the head group, the length of the alkyl tails, and the packing of the lipids. ${ }^{15}$ For example, it was demonstrated that the permeability of phospholipid bilayers in their fluidic phase $\left(T>T_{\mathrm{m}}\right)$ to protons, potassium ions, water, urea, and glycerol, decreases with chain length (from 14 to 24 carbon atoms) for unsaturated lipids; for instance, proton permeation was reduced from $K_{i}=$ $1.3 \times 10^{-2} \mathrm{~cm} \mathrm{~s}^{-1}$ to $4.9 \times 10^{-5} \mathrm{~cm} \mathrm{~s}^{-1}$, when increasing membrane thickness from $2.0 \mathrm{~nm}$ to $3.8 \mathrm{~nm} .{ }^{41}$

The permeability coefficient $(P)$ varies per molecule and is dependent on the charge, polarity, size and molecular mass of the molecule in motion. If $\Delta G$ is the change in free Gibbs enthalpy $\left(\mathrm{kJ} \mathrm{mol}^{-1}\right)$ involved when moving a solute from the bulk aqueous phase to the hydrophobic core of the membrane, one can estimate whether a molecule can cross the lipid bilayer $(\Delta G \leq 0)$ by using $\log P$ as a guideline (Fig. 10). In general, very hydrophobic molecules dissolve well inside the hydrophobic lipid bilayers and accumulate there, such as DPPC and cholesterol $(\log P \gg 0$, Fig. 10c). Amphiphilic small molecules characterised 

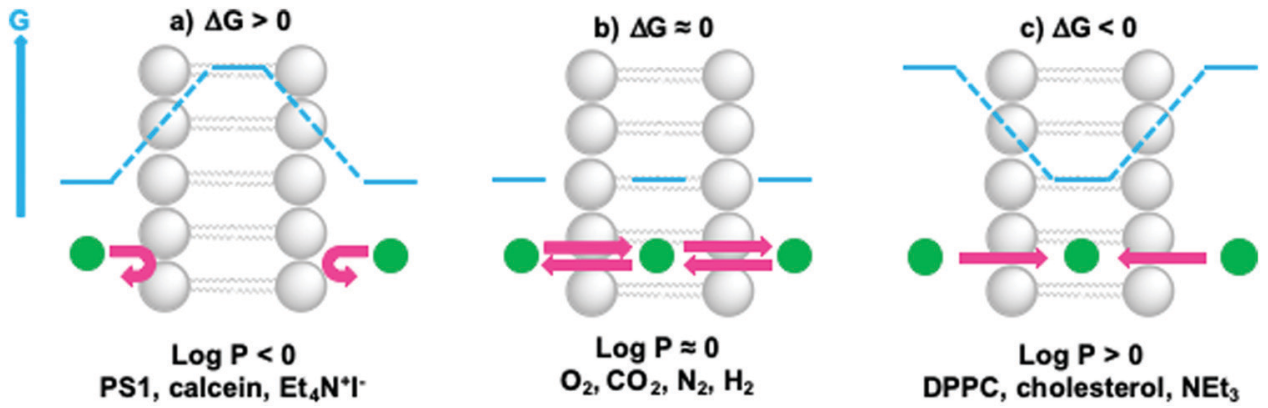

Fig. 10 Membrane permeability and residence time of ions and molecules depends on their hydrophilicity. When (a) $\log P \ll 0, \Delta G>0$ and molecules cannot cross or enter the lipid bilayer; when (b) $\log P \approx 0, \Delta G \approx 0$, molecules can redistribute between the aqueous phase and the hydrophobic core of the lipid bilayer, and cross the membrane; when (c) $\log P \gg 0, \Delta G<0$ and molecules prefer to reside within the lipid bilayer. Actual log $P$ values can be found in references ${ }^{16,21,42}$ or can be obtained by using $\log P$ predictors such as miLogP2.2 (http://www.molinspiration.com). For example, log $P=+7.62$ for cholesterol, $\log P=+0.09$ for $\mathrm{O}_{2}, \log P=+0.02$ for $\mathrm{CO}_{2}, \log P=+0.02$ for $\mathrm{N}_{2}$, and $\log P=+0.16$ for $\mathrm{H}_{2}$.

by $\log P \approx 0$, which includes gas molecules relevant to artificial photosynthesis $\left(\mathrm{O}_{2}, \mathrm{CO}_{2}, \mathrm{~N}_{2}\right.$, or $\left.\mathrm{H}_{2}\right)$, can either accumulate or diffuse across the lipid bilayer (Fig. 10b). Neutral polar molecules, such as $\mathrm{H}_{2} \mathrm{O}$, can also diffuse through a lipid bilayer albeit much slower than small hydrophobic molecules, because they prefer to be solubilised by water. ${ }^{40}$ On the other hand, hydrophilic, charged molecules such as PS1 or calcein characterised by a $\log P \ll 0$, are poorly permeable to the lipid membrane because it is too costly to dissolve them in the hydrophobic core of the membrane (Fig. 10a). ${ }^{21,42}$ Similarly, ions, protons, and electrons, are poorly permeable to the membrane and therefore their transfer across a lipid bilayer has to be facilitated by other means such as membrane channels or ion pumps (see Section 2.3 for more details). To illustrate these points with some numbers, the permeability coefficients through lecithin-based lipid bilayers are generally in the order of $10^{-3}$ to $10^{-4} \mathrm{~cm} \mathrm{~s}^{-1}$ for $\mathrm{H}_{2} \mathrm{O}, 10^{-11}$ to $10^{-12} \mathrm{~cm} \mathrm{~s}^{-1}$ for anions, and $10^{-12}$ to $10^{-14} \mathrm{~cm} \mathrm{~s}^{-1}$ for cations that are commonly utilised such as $\mathrm{Na}^{+} \cdot{ }^{40}$ In some other cases certain charged molecules, such as the charge carrier cetyl methyl viologen (CMV), have a different mechanism to cross the lipid bilayer. The stable and twofold positively charged form, $\mathrm{CMV}^{2+}$, is water soluble and barely penetrates the lipid bilayer. Likewise, the singly reduced species $\mathrm{CMV}^{+}$is still positively charged and anticipated to rest in the aqueous phase. However, upon a disproportionation reaction two singly-charged $\mathrm{CMV}^{+}$molecules can generate a $\mathrm{CMV}^{2+}$ and a neutral $\mathrm{CMV}^{0}$ molecule, which can freely diffuse across a lipid bilayer. ${ }^{43}$

Although membrane leakage has mainly been studied for liposome-based drug delivery, it is also an important aspect in artificial photosynthesis applications, especially when preparing dissymmetrical liposomes for transmembrane electron, proton, or ion transfer (see Section 2.6). For such systems, the interior of the liposome contains a chemical that is absent from the outside bulk solution, or vice-versa. To demonstrate transmembrane electron- or proton-transfer, both sides of the membrane should not exchange molecules by membrane leakage. For example, transmembrane electron transfer was initially reported for a system containing the charge carrier $\mathbf{M V}^{\mathbf{2}}$ (see Fig. 7a) in the inner aqueous compartment of sodium dihexadecyl phosphate
(DHP) vesicles, whereas the PS1 and the sacrificial ED (EDTA) were located in the bulk. ${ }^{7}$ However, several studies pointed to the photoredox reaction between photoexcited $\mathbf{P S 1}$ and $\mathbf{M V}^{2+}$ actually occurring in the bulk, due to leakage of both $\mathbf{M V}^{{ }^{++}}$and PS1 across the DHP bilayer. ${ }^{7}$

As a side note, the membrane permeability of molecules does not depend only on their $\log P$, as water-soluble compounds may also permeate membranes via holes or ruptures in the membrane. The lipid composition, for example, was shown to play an important role in membrane leakage. Liposomes prepared from the unsaturated lipid DOPC $\left(T_{\mathrm{m}}=-17^{\circ} \mathrm{C}\right)$ have a larger release rate at room temperature for water-soluble molecules than liposomes prepared from the saturated lipid DPPC $\left(T_{\mathrm{m}}=+41{ }^{\circ} \mathrm{C}\right) .{ }^{42}$ Generally, vesicles prepared from saturated lipids with longer alkyl chain lengths pack more tightly, possess a high gel-to-liquid crystalline transition temperature $\left(T_{\mathrm{m}}\right)$, and retain entrapped solutes better than unsaturated lipids and shorter alkyl tail lengths who have a less rigid lipid bilayer assembly. Furthermore, addition of cholesterol to the lipid composition leads to increased lipid bilayer stability and less membrane leakage. Last but not least, liposomes are leakier near their phase transition temperature $T_{\mathrm{m}}$ due to the co-existence of gel-phase and liquid-phase domains within the membrane, which generate grain junction through which polar molecules may diffuse. As a practical consequence, vesicles constructed from DMPC, which has a transition temperature $T_{\mathrm{m}}=+23{ }^{\circ} \mathrm{C}$ that is near room temperature, are typically leakier in room-temperature experiments, compared to DPPC-based liposomes which have $T_{\mathrm{m}}$ significantly higher than $20-25{ }^{\circ} \mathrm{C}$ (Fig. 11).$^{44}$ Photocatalytic membranes based on DMPC should hence be studied at lower or higher temperature than room temperature.

2.4.2 Membrane leakage: experimental approaches. Leakage can be quantified experimentally using self-quenching dyes, such as calcein, 5(6)-carboxyfluorescein and sulforhodamine B. A typical membrane leakage experiment consists in encapsulating one of these water-soluble dyes at high concentration inside liposomes, which causes low emission levels due to self-quenching (Fig. 12). Upon release of the water-soluble dye into the outside bulk solution, i.e., upon membrane leakage, the emission intensity 


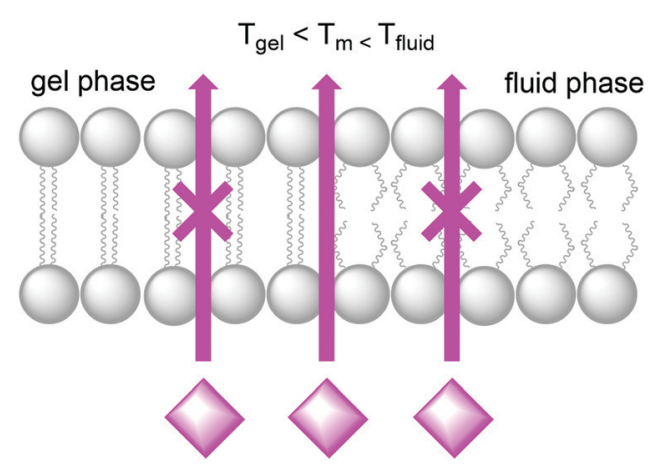

Fig. 11 Water-soluble substrate (pink diamonds) crosses membranes more easily near the transition temperature $T_{m}$ due to permeable grain junction between fluid phase and gel phase domains.

increases because the dye outside the liposome is not selfquenched anymore. At the end of the experiment, a detergent such as Triton-X-100 is added to the liposome sample, resulting in the complete disruption of the liposome, full release of the dye, and subsequent formation of micelles containing both the liposomal lipids, the detergent, and the dye. This point is taken as reference (unquenched) emission with $100 \%$ dye leakage. It should be noted that all self-quenching dyes used for such experiments are negatively charged and may self-assemble with positively charged components of the membrane. Currently, there is a lack of cationic self-quenching dyes for membrane leakage experiments.

An alternative method is to use dye-quencher pairs, such as 8-aminonapthalene-1,3,6-trisulfonic acid with $p$-xylene-bis-pyridinium bromide (ANTS/DPX), and fluorescent enhancement pairs, like $\mathrm{Tb}^{3+}$ and dipicolinic acid $\left(\mathrm{Tb}^{3+} / \mathrm{DPA}\right) .^{45}$ For ANTS/DPX, both molecules are co-encapsulated in the vesicle and quench each other; upon disruption of the lipid bilayer by a detergent luminescence increases because quenching becomes smaller. The latter case involves one of the only positively charged probes used for leakage tests. Here, the weakly luminescent $\mathrm{Tb}^{3+}$ ions leaks from the interior of the liposome and coordinates to DPA present in the bulk outside solution, leading to the formation of a highly luminescent $\left[\mathrm{Tb}(\mathrm{DPA})_{3}\right]$ complex. ${ }^{45}$
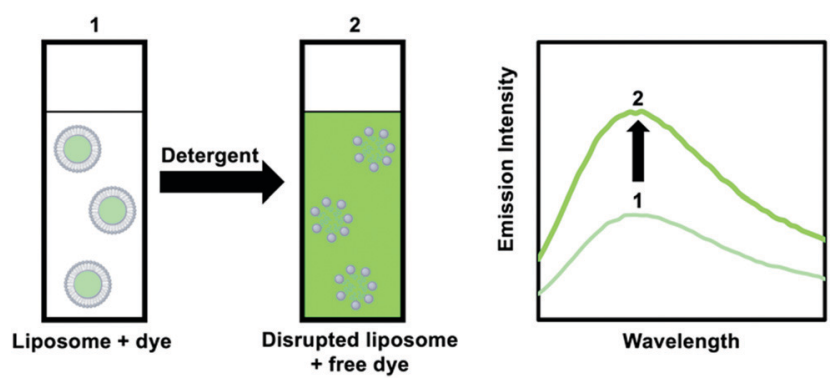

Fig. 12 Addition of a detergent to a liposome solution with an encapsulated and weakly luminescent dye results in the release of the dye and enhanced emission intensity.

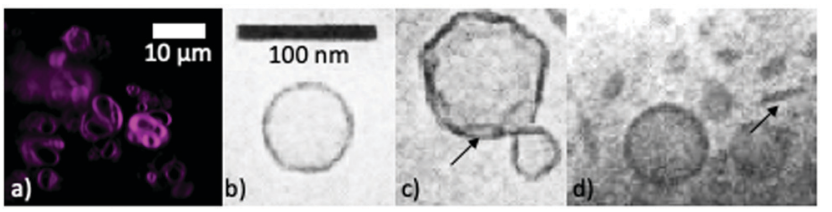

Fig. 13 (a) Confocal microscopy image of a luminescent dye embedded in the lipid bilayer of giant DMPC vesicles. Figure adapted from ref. 39. CryoTEM images of (b) extruded DMPC $\left(T_{m}=23^{\circ} \mathrm{C}\right)$ vesicles vitrified from $40{ }^{\circ} \mathrm{C}$, (c) extruded DPPC $\left(T_{\mathrm{m}}=41^{\circ} \mathrm{C}\right)$ vesicles vitrified from $25^{\circ} \mathrm{C}$, and (d) sonicated DLPA $\left(T_{\mathrm{m}}=31^{\circ} \mathrm{C}\right)$ vesicles vitrified from $18^{\circ} \mathrm{C}$. Adapted with permission from M. Andersson, L. Hammarström and K. Edwards, J. Phys. Chem., 1995, 99, 14531-14538. Copyright (1995) American Chemical Society. ${ }^{34}$

\subsection{Shape of vesicles}

Vesicles exist in different sizes and shapes and their preparation is described in Section 3.1. Vesicles that are visible with a microscope should have a micrometre diameter and are called "giant" vesicles (Fig. 13a). Vesicles that are smaller than $1 \mu \mathrm{m}$ are either called "large" or "small" vesicles (Fig. 13b). Both types can be unilamellar (only one bilayer) or multilamellar (several bilayers) with multiple membranes forming an onionlike "vesicle within a vesicle". Liposomes are spherical vesicles made of at least one unilamellar membrane. In principle, the larger the vesicle becomes, the more shapes are possible, which can be seen in the confocal image of membrane-stained giant vesicles in Fig. 13a. Apart from spheres, ellipsoids, tubes and other shapes have also been described.

The morphology of the lipid bilayer of liposomes depends on the lipid head group, the length of the hydrocarbon tails, the size of the liposomes, the transition temperature of the lipid, and the preparation methodology. Decreasing the temperature of a liposome solution to values below $T_{\mathrm{m}}$, may result in faceted vesicles (Fig. 13c) or the creation of open bilayer fragments (Fig. 13d) instead of smooth round vesicles, as can be experimentally visualised by cryogenic transmission electron microscopy (CryoTEM, see Fig. 13b-d). ${ }^{34}$ These morphological changes have an effect on photoredox reactions, such as the reduction of CMV by dithionite $\left(\mathrm{S}_{2} \mathrm{O}_{4}{ }^{2-}\right) \cdot{ }^{34}$ In the case of smooth vesicles, monoexponential reduction kinetics can be observed, whereas the presence of bilayer irregularities and non-vesicular structures can lead to double-exponential reduction kinetics. ${ }^{34}$ Therefore, in order to ease the study of complex photocatalytic reactions occurring at the membrane-water interface, such as electron, proton, or ion transport across a lipid bilayer, ${ }^{34}$ one should aim to use regular unilamellar vesicles either in the fluid or the gel phase.

\subsection{Transport of electrons, protons, and ions across a lipid bilayer}

Biological transmembrane electron transfer takes place in cell compartments of photosynthetic and respiratory organelles to allow for redox reactions to occur and to generate an imbalance in electrochemical potential across the membrane. This imbalance is translated into a proton motive force that is ultimately used as an energy source to drive endergonic transformations such as the 
generation of ATP by the membrane-embedded ATPase. There are also examples for ion transfer that act without proton motive forces, such as simple passive transmembrane protein channels such as gramicidin A, aquaporin, the prokaryotic potassium channels of streptomyces A, or even artificial ion channels or transporters. In this review, we only consider the light-induced transport of electrons, protons, and ions across lipid bilayers for artificial photosynthesis.

Electron transfer through a lipid bilayer cannot generally occur by direct tunnelling between reactants on either side of the membrane as the thickness of the hydrophobic core of typically ca. 30-35 $\AA$ is too high to allow direct charge transfer at reasonable rate. The electron transfer rate, $k$, is given by eqn (13):

$$
k=A \cdot \mathrm{e}^{-\beta r}
$$

where $A$ is the exponential prefactor, $\beta$ the attenuation factor (in $\AA^{-1}$ ), and $r$ is the distance (in $\AA$ ). $\beta$ is typically around $1.0 \AA^{-1}, 46$ which would make even an activationless reaction proceed on a time scale as slow as minutes to hours. Instead, the electrons are typically carried across the membrane by small molecules, or transferred by hopping in several shorter steps via redox centres located inside the hydrophobic part of the membrane as it occurs in thylakoid membranes.

To mimic natural transmembrane redox processes the following strategies are possible:

(a) To incorporate biologic components into the lipid bilayer such as natural reaction centres and membrane-bound electron transfer proteins (Fig. 14a-e).

(b) To synthesise transmembrane molecules spanning the lipid bilayer, such as rigid molecular triads or supramolecular assemblies of electron conduits (Fig. 14f-h).

(c) To organise the diffusion of molecular charge carriers embedded within the lipid bilayer of artificial membranes (Fig. 14e, f and i).

Although many examples have been reported, ${ }^{7}$ we only present here a selection of examples to illustrate the three strategies discussed above.

Strategy a is best represented by a semiartificial compartmentalised liposome system (Fig. 14d) that used the protoncoupled icosa-haem transmembrane electron transfer protein complex, MtrCAB (analogous to S. Baltica OS185's structure shown in Fig. 7c). ${ }^{5}$ MtrCAB is a cytochrome-type protein complex found in Shewanella oneidensis MR-1 bacteria, where it acts as a natural transporter of electrons to external minerals. In the reported system, phosphonated ruthenium(II) tris-bipyridine $\left(\left[\mathrm{Ru}(\mathrm{bpy})_{2}\left(4,4^{\prime}-\left(\mathrm{PO}_{3} \mathrm{H}_{2}\right)_{2} \mathrm{bpy}\right)\right]^{2+}\right.$, RuP $)$ photosensitised titanium oxide nanoparticles $\left(\mathbf{R u P}-\mathrm{TiO}_{2}\right)$, and carbon nanodots were employed as synthetic light harvesters placed outside the liposome. To demonstrate spectroscopically transmembrane electron transfer with MtrCAB embedded in a synthetic liposome, the redox active dye, reactive red 120 (RR120), was encapsulated inside the liposome and showed the expected bleaching of the band at $539 \mathrm{~nm}$ upon photo-reduction. The sacrificial electron donor EDTA regenerated the hole left in the light-harvesting nanoparticles. The rate limiting step was identified as the electron transfer from the light-harvesting nanoparticles to the
MtrCAB electron relay. ${ }^{5}$ In a similar example, a re-purposed photosynthetic reaction centre, isolated from $R$. sphaeroides bacteria, was embedded in the membrane of a giant unilamellar vesicle with the desired physiological orientation by following a droplet transfer synthetic method (Fig. 14e). ${ }^{47}$ With this protocol roughly $90 \%$ of the photosynthetic reaction centre was facing the aqueous bulk solution and, thus, $90 \%$ of the quinone sites $\left(\mathbf{Q}_{\mathbf{B}}\right)$ were facing the inner space of the lipid bilayer, thus creating an artificial lumen that enabled the generation of a light-driven proton gradient across the membrane. This semiartificial vesicle was used in combination with the water-soluble ED cytochrome $c_{2}$, the natural electron donor to this reaction centre, as well as ferrocyanide $\left(\left[\mathrm{Fe}(\mathrm{CN})_{6}\right]^{4-}\right)$ in the bulk; on the other hand, the quinone on the inner monolayer of the vesicle was used as final EA. Under constant red-light irradiation $(\lambda=865 \mathrm{~nm})$ the repurposed reaction centres converted light energy to the energy of a proton gradient across the lipid bilayer at about $0.061 \mathrm{pH} \mathrm{min}^{-1}$, which is equivalent to a proton motive force of $3.6 \mathrm{mV} \mathrm{min}^{-1}$. Hence, the reduction and subsequent protonation of the quinone within the membrane induced a $\mathrm{pH}$ gradient in addition to the electrochemical potential. ${ }^{47}$

In strategy $b$, fully synthetic examples were developed to couple transmembrane electron transfer to transmembrane proton transfer. A prominent example is the synthetic molecular triad (Q-P-C, Fig. 14f) consisting of a naphthoquinone moiety as electron acceptor (Q), the PS tetraarylporphyrin (P), and a carotenoid as electron donor $(\mathbf{C})$. This triad was embedded into a lipid bilayer and transported electrons across a membrane upon light irradiation. ${ }^{33}$ Control titration studies showed that the majority of triads were arranged with their $\mathbf{Q}$ ends facing the aqueous bulk, while the $\mathbf{C}$ moiety spanned the lipid bilayer towards the inner aqueous compartment. Irradiation of the triad with laser pulses at $430 \mathrm{~nm}$ excited the porphyrin group, which results in the formation of a charge separated species $\mathbf{Q}^{-}-\mathbf{P}-\mathbf{C}^{+}$ that could be detected by the transient absorbance of the carotenoid radical cation at $930 \mathrm{~nm}$. This biradical was generated with a quantum yield of 0.1 and had a lifetime of $60 \mathrm{~ns}$ within the lipid bilayer in the presence of freely diffusing quinones $\left(\mathbf{Q}_{\mathbf{s}}\right)$, which established a reduction potential near the outer surface of the bilayer and an oxidation potential near its inner surface. In response to that, $\mathbf{Q}_{\mathbf{s}}$ accepted an electron from the $\mathbf{Q}^{-}$part of the triad generating a reduced and anionic quinone that got protonated and hence transferred protons across the bilayer by electron transfer to the oxidised $\mathbf{C}^{-}$part of the triad, with an overall quantum yield of $0.004 .^{33}$ This process turned over during light irradiation, generating a $\mathrm{pH}$ gradient between the inner compartment and the bulk. ${ }^{33}$ Ultimately, in a follow-up study, the proton motive force generated by this artificial liposome was coupled to a membrane-bound ATP synthase complex in the same lipid bilayer, which upon irradiation resulted in the synthesis of ATP with an initial quantum yield at low-light irradiation of more than 0.07 for the whole process within the new assembly. ${ }^{35}$ In another example, $p$-octiphenyl rods were used to create a helical tetrameric supramolecular assembly with $\pi$-stacked blue and red-fluorescent naphthalene diimides spanning the lipid bilayer of a liposome, which also contained freely diffusing quinones as EA (Fig. 14g). The functionalised liposomes were 
a) Assemble of (semi)artificial ET components

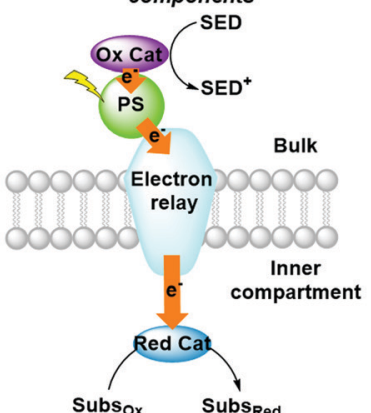

d) Assemble of transmembrane cytochrome proteins for ET

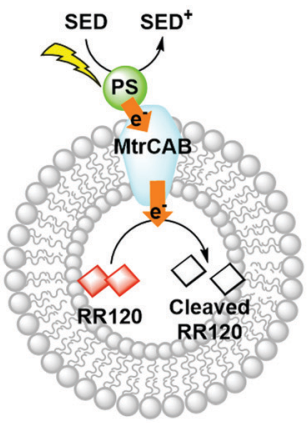

g) Supramolecular $\pi$-stacked
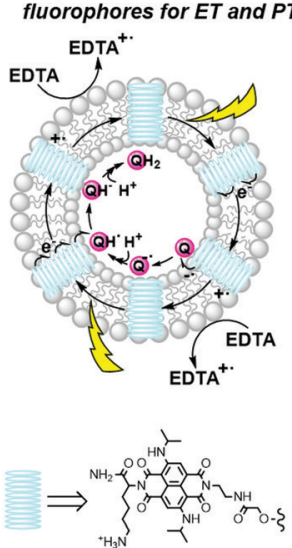

b) Assemble of (semi)artificial ion channels

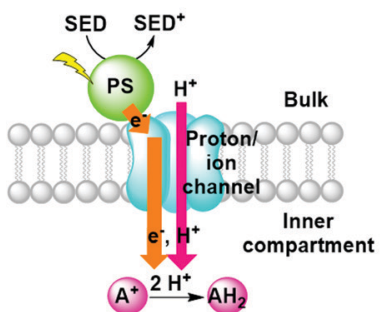

e) Repurposing natural RC and PT

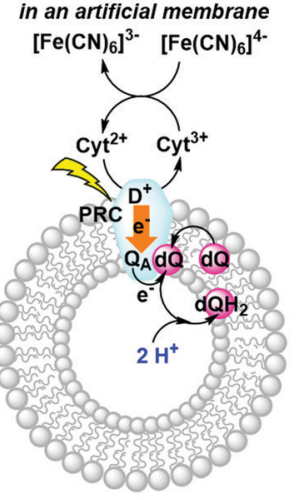

h)
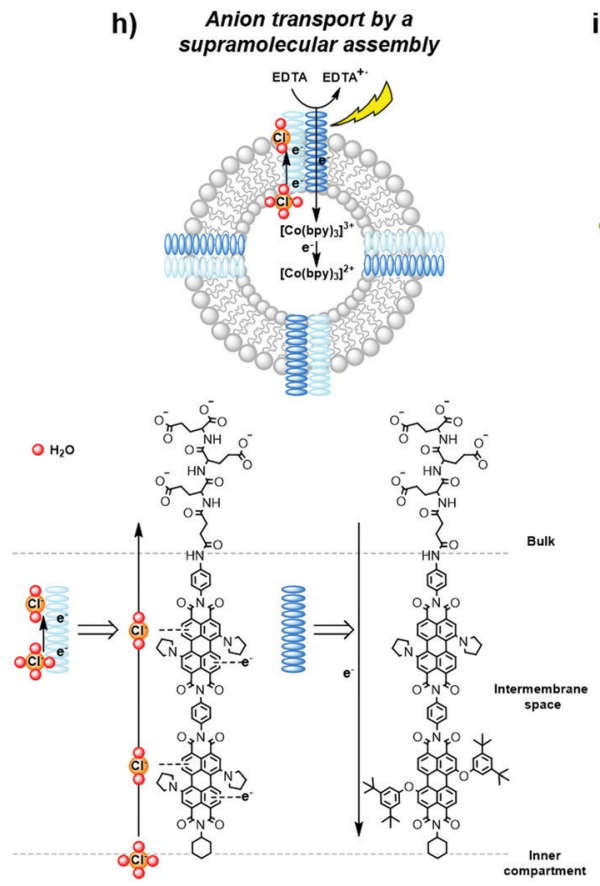

c) Assemble of (semi)artificial ET coupled to PT components

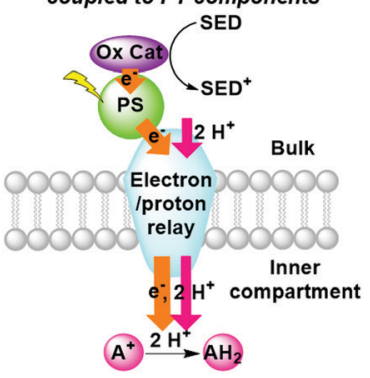

f) Assemble of transmembrane triads for ET-PT vesicle $\mathrm{H}^{+}$
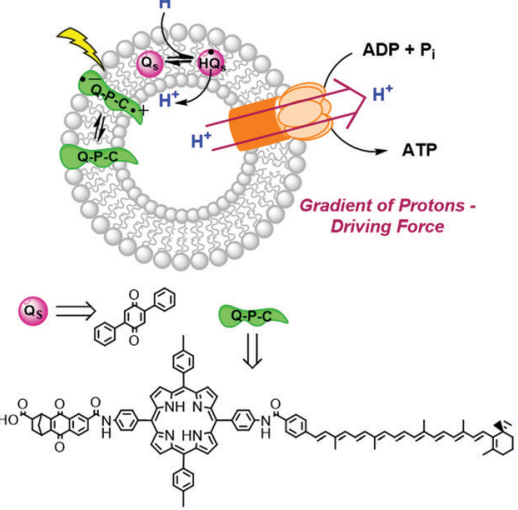

i) Free diffusion ET molecule and assembled $P S$ vesicle

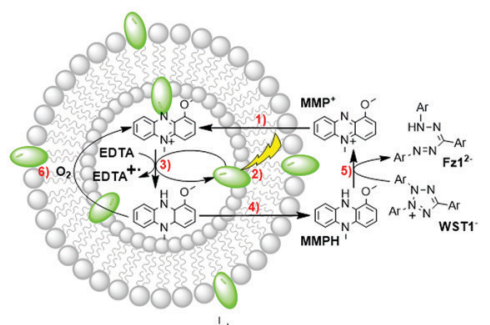

Fig. 14 General strategies for the development of (semi)artificial liposome systems $(a-c)$ and selected examples (d-i) for proton and electron transfer across lipid bilayers. Assemblies of (a) (semi)artificial electron transfer (ET) components, (b) (semi)artificial ET coupled to proton transfer (PT) components across the membrane, (c) (semi)artificial ion channels, (d) transmembrane cytochrome (MtrCAB) proteins for ET, ${ }^{5}$ (e) repurposed natural photosynthetic reaction centres $(\mathrm{PRC})$ and proton transfer components in an artificial membrane, ${ }^{47}$ (f) transmembrane quinone-porphyrin-carotenoid triad $(\mathrm{Q}-\mathrm{P}-\mathrm{C})$ for coupled ET-PT across the membrane, ${ }^{33,35}(\mathrm{~g})$ supramolecular $\pi$-stacked fluorophores for coupled ET and PT, ${ }^{48}$ (h) supramolecular transmembrane rods for electron and anion transport, ${ }^{49}$ and (i) membrane-embedded PSs combined with a diffusion-based ET molecule. ${ }^{26}$ Ox. Cat. $=$ oxidation catalyst;

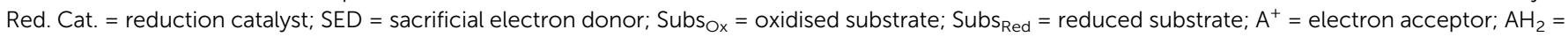
reduced and protonated electron acceptor; $Q_{s}$ and $d Q=$ membrane-soluble quinone (Fig. 7), $D^{+}=$donor; $Q_{A}=$ quinone $A$ from the isolated reaction centre. 
immersed in an aqueous solution containing EDTA as ED in the bulk. The irradiation of the $\pi$-stacked dyes with visible light generated a fast electron transport across the interacting chromophores until the electron was finally accepted by the quinone in the inner aqueous compartment, which was accompanied by proton transfer to the quinone. The long-lived charge separated state across the membrane was hence translated into an oxidation potential near the outer surface of the membrane and a reduction potential near the inner surface, as well as a proton gradient across the membrane. ${ }^{48}$ In a third and last example of coupled electron and ion transmembrane transfer, rigid oligo( $p$-phenylene)- $N, N$-perylenediimide rods were embedded in egg yolk phosphatidylcholine based vesicles (Fig. 14h). ${ }^{49}$ These rods were able to perform photoinduced transmembrane electron transfer from EDTA in the bulk to the electron-accepting charge carrier $\left[\mathrm{Co}(\mathrm{bpy})_{3}\right]^{2+}$ in the inner aqueous phase, while simultaneously transporting $\mathrm{Cl}^{-}, \mathrm{OH}^{-}$or $\mathrm{SO}_{4}{ }^{2-}$ anions, in the reverse direction. Interestingly, the rods failed to transport cations at all and its selectivity for anion transport was thus proposed to result from anion- $\pi$ interactions along the $\pi$-acidic environments of the oligo-aromatic rods. ${ }^{49}$

Finally, in strategy $c$ the membrane-soluble molecular charge carrier $\mathbf{M M P}^{+}$was employed for unidirectional transmembrane electron transfer in liposomes. In this system, PS5 was embedded in the membrane, EDTA was placed in the inner space of the liposome as ED, and 2-(4-iodophenyl)-3-(4-nitrophenyl)-5-(2,4disulfophenyl)-2 $\mathrm{H}$-tetrazolium anion (WST1 ${ }^{-}$) was finally added in the exterior aqueous bulk solution as EA (Fig. 14i). ${ }^{26}$ In an anaerobic atmosphere, $\mathbf{M M P}^{+}$catalytically transported photoelectrons across the membrane without limiting the overall reaction rate. In such conditions late charge recombination of reduced WST1 $^{-}$with $\mathrm{ED}^{+}$, as well as quenching of PS5* excited states by the reduced $\mathbf{W S T 1}^{-}$were avoided by their physical separation with the liposomal membrane. However, as soon as oxygen was introduced in the system transmembrane photoelectron transfer stopped occurring, because MMPH, the reduced form of $\mathbf{M M P}^{+}$, reacted with $\mathrm{O}_{2}$ faster than with $\mathbf{W S T}^{-}$. This limitation shows how tricky it is to avoid charge recombination even at a late stage of the photocatalytic cycle.

\section{The water membrane interface}

\subsection{Preparation of liposomes and generation of confined aqueous reaction spaces}

Vesicles provide three different reaction spaces: the inner aqueous compartment, the hydrophobic core of the membrane, and the outer aqueous bulk solution. These three spaces can be addressed separately, loaded with different photocatalytic components, and characterised via different experimental techniques. Table S1 (ESI $\dagger$ ) lists these techniques and which information one can obtain from them.

By self-assembly of lipid bilayers into vesicles a high local concentration of reactants can be achieved in the bilayer or by the encapsulation of the substrates in the inner aqueous compartment, even upon dilution of the vesicle solution. As an example, a vesicle with a diameter of $100 \mathrm{~nm}$ has an internal volume of approximately $10^{-13} \mu \mathrm{L}$. Confinement within the vesicle can be important because the local concentration of water soluble substrates and products influences reaction dynamics in water and at the interface of the membrane. ${ }^{1,9}$ Furthermore, the hydrophilic products from water oxidation and $\mathrm{CO}_{2}$ reduction, such as protons and formate, respectively, might affect membrane stability due to changes of the osmotic pressure. For example, when oxidising two water molecules to one molecule of $\mathrm{O}_{2}$ that diffuses away, four protons are generated as well, which changes the $\mathrm{pH}$ and osmotic pressure.

For unidirectional electron transfer through membranes, it can be particularly appealing to differentiate the inner and outer reaction spaces in vesicles, for example by loading the interior space of the vesicles with functional molecules or substrate, or by adding after liposome preparation, compounds that will only be present on the outside. Among all methods to prepare liposomes, a standard one is depicted in Fig. 15. Firstly,

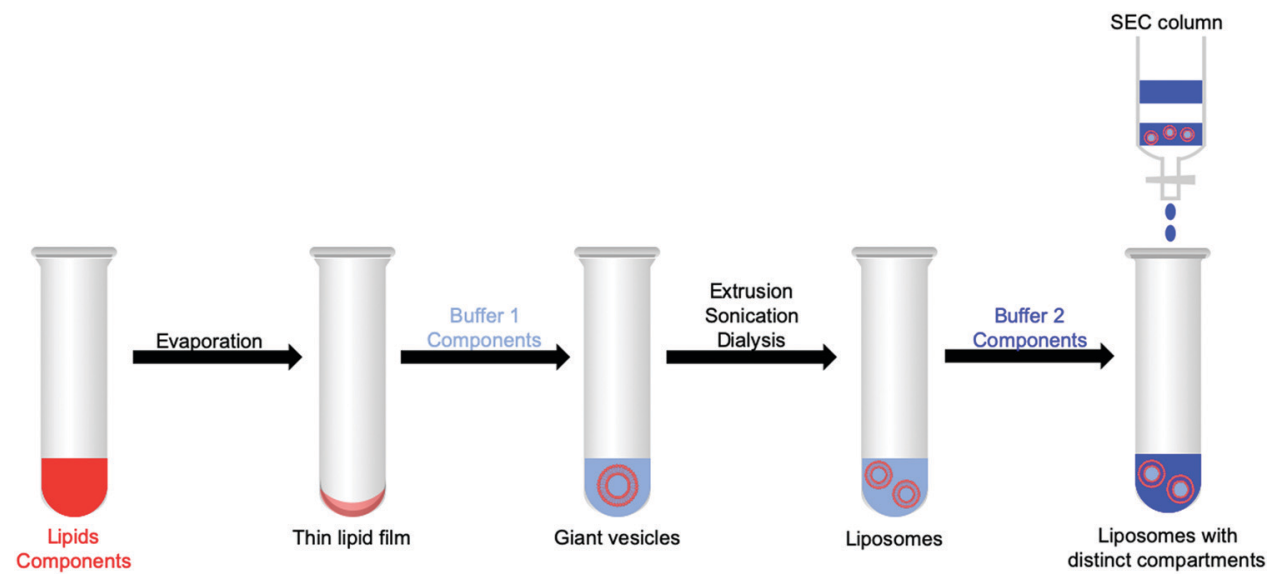

Fig. 15 Method for the preparation of liposomes with distinct compartments that are functionalised with active components for artificial photosynthesis. $\mathrm{SEC}=$ size exclusion chromatography. Membrane soluble components are shown in red, whereas water-soluble components are shown in light or dark blue. Light blue shows Buffer 1, dark blue Buffer 2 (see text). 
a solution of lipids and membrane-soluble components, such as PSs and catalysts (see Section 1.3) dissolved in organic solvents, is transferred to a glass tube or flask. Subsequently, the organic solvents are removed under reduced pressure, resulting in the formation of a thin lipid film on the glass wall of the tube or flask. In the next step, the vacuum dried thin lipid film is hydrated using an aqueous buffer containing water-soluble components, such as sacrificial reagents or charge carriers (Buffer 1). Several freeze-thaw cycles (in some cases also sonication) induce the formation of giant multilamellar vesicles (diameter $>1 \mu \mathrm{m}$ ). To obtain liposomes (diameter $<1 \mu \mathrm{m}$ ), extrusion with a suitable pore size filter, sonication, or dialysis, can be performed. Extrusion typically provides relatively narrow hydrodynamic diameter distributions characterised by low polydispersity index (PDI) in DLS or cryo-TEM analysis (PDI $<0.1$ ). At this stage both sides of the membrane are identical; however, the bulk solution can be replaced by a second and different aqueous buffer solution (Buffer 2) containing water-soluble components that are absent from the interior; using size exclusion chromatography (SEC), it is then possible to isolate the liposomes filled with Buffer 1 from excess Buffer 1. Dissymmetrical liposomes are then obtained, filled with Buffer 1 and surrounded with Buffer 2. As mentioned above, with such liposomes it is critical to use two Buffers 1 and 2 with identical osmotic pressure, as measured by an osmometer. Otherwise, differences in osmotic pressure on both sides of the membrane lead to the uptake or release of water from the inner aqueous compartment, which may make the membrane leaky (see Section 2.3).

\subsection{Properties of the interface}

3.2.1 Local concentrations at self-assembled lipid membranes. For efficient photocatalysis, managing local concentrations of active components, protons fluxes, and redox potentials, are crucial. Lipid bilayers typically enhance local concentrations when PS and catalyst molecules are co-embedded in the lipid bilayer, resulting in closer proximity of these components, compared to homogeneous solution with identical bulk concentrations. An example was reported for photocatalytic water oxidation (Fig. 16), where PS1 and a non-alkylated WOC in homogeneous solution was compared with co-embedded PS2 and WOC in DMPC based liposomes, using $\mathrm{Na}_{2} \mathrm{~S}_{2} \mathrm{O}_{8}$ as sacrificial EA. ${ }^{23}$ The photochemical reaction involved three identified steps in both photocatalytic systems: first, light induced electron transfer between the PS and the EA (rate $r_{1}$ ), second, electron transfer between the oxidised PS and the catalyst (rate $r_{2}$ ), and finally, redox catalysis (rate $r_{3}$ ). In homogeneous conditions, $r_{1}$ and $r_{2}$ are limited by diffusional processes, which resulted in concentration-dependent electron transfer steps 1 and $2 .^{23}$ Due to the high concentration of the EA $(\mathrm{mM})$, the rate-limiting step (RDS) was found to be step 2, as the catalyst concentration was much lower $(\mu \mathrm{M})$. On the other hand, when PS and the water oxidation catalyst were embedded in the membrane the electron transfer rate between WOC and $\mathbf{P S 2}^{+}$, i.e. step 2 , became much faster and stopped being rate determining. Instead, step 1 became rate limiting. ${ }^{6,23}$ This specific change in RDS from step 2 to step 1 significantly improved the overall photocatalytic reaction, notably because the PS decomposition was partly prevented by the lower concentration of $\mathbf{P S 2}^{+}$. This form of decomposition is an important limiting factor in water oxidation systems employing ruthenium polypyridine-based PSs. ${ }^{23}$ Using UV-Vis spectroscopy, it was found that the rate of oxidative quenching of photoexcited PS2 by $\mathrm{Na}_{2} \mathrm{~S}_{2} \mathrm{O}_{8}$ in liposomes was at least one order of magnitude lower than with the homogeneous analogue PS1 in homogeneous conditions. Namely, PS2 was either regenerated via electron transfer from the catalyst or decomposed in the oxidised state $\mathbf{P S 2}^{+}$. At decreasing concentrations of WOC the formation of $\mathbf{P S}^{+}$increased with concomitant decomposition, highlighting that electron transfer from WOC
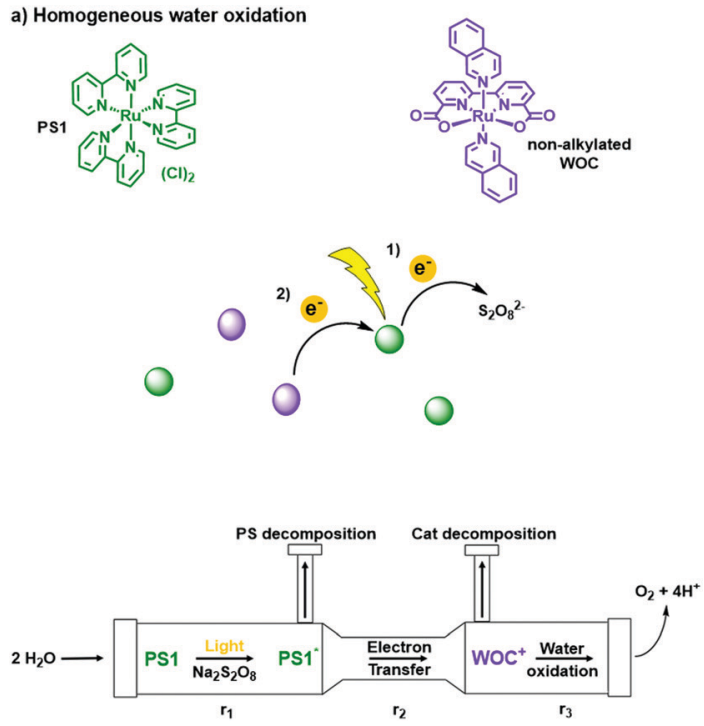

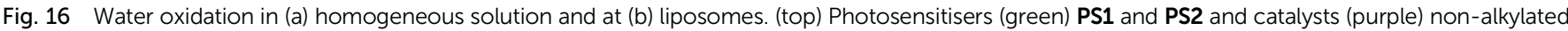

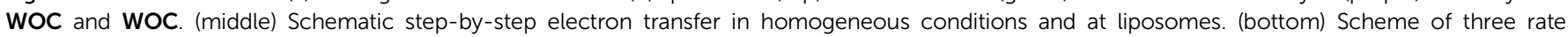
determining steps (RDS) in photocatalytic water oxidation under homogeneous and liposome conditions. Figure adapted with permission from ref. 50.
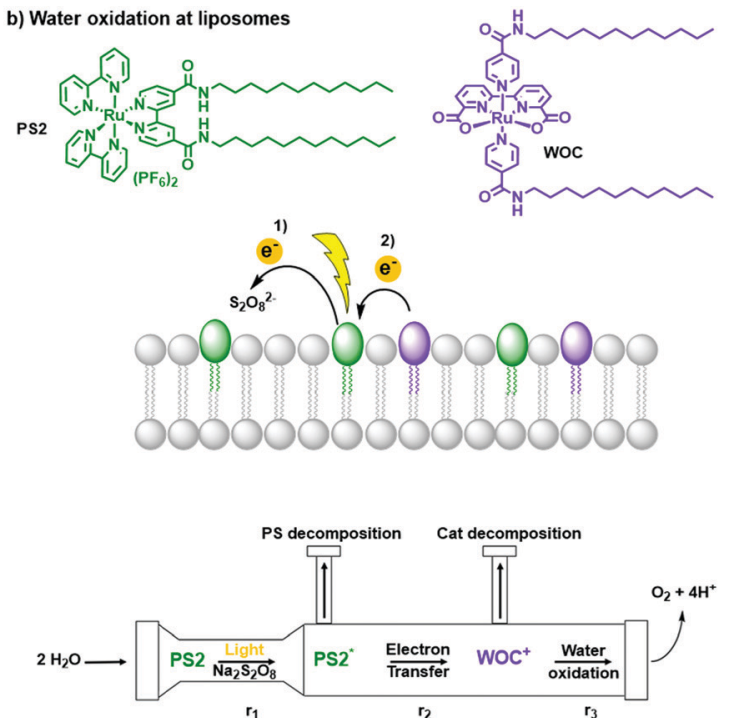
to PS2 $^{+}$and catalytic oxidation of water is fast enough to maintain the concentration of the sensitive $\mathbf{P S}^{+}$at concentrations below the detection limit. ${ }^{23}$ These results also highlight that the stability of a photocatalytic system highly depends on kinetics, and notably on the synergistic performances of the four components (light, PS, cat, EA) working as a team, and not only on the intrinsic stability (and even photostability) of its individual components. $^{23}$

Another surface concentration effect that should be noted is related to protons; proton management is indeed important, as most redox reactions in artificial photosynthesis are coupled to proton transfer (PCET, see for example eqn (1)-(6)). At lipid bilayer interfaces the protonation-deprotonation dynamics are different than in homogeneous solution. This protonationdeprotonation equilibrium is described by the acidity constant, $\mathrm{p} K_{\mathrm{a}}$. The $\mathrm{p} K_{\mathrm{a}}$ of a proton-donating or accepting group at the bilayer-water interface differs from the value in bulk water solutions, essentially due to different surface polarities (dielectric constant), and the electrostatic enhancement or reduction of proton concentration at the bilayer interface due to surface charge changes. For example, while the acidity constants of most lipids are tabulated, ${ }^{15}$ it should be noted that $\mathrm{p} K_{\mathrm{a}}$ values at a membrane can shift by up to $\Delta \mathrm{p} K_{\mathrm{a}}=2.5 .^{15}$

3.2.2 Ion "belts" around charged membrane surfaces. The effect of counterions on the properties of lipid membranes is often forgotten while they may result in changes in two-dimensional diffusion properties and electron transfer kinetics between membrane-bound species as a result notably of phase transitions. For example, cations with large charge densities such as $\mathrm{Ca}^{2+}$ and $\mathrm{Li}^{+}$adsorb to zwitterionic lipid membranes, which can induce rigidification of the membrane and a stabilisation of their gelphase. ${ }^{38}$ This phenomenon was investigated using $\mathrm{NaCl}, \mathrm{KCl}$ and $\mathrm{CsCl}$ with liposomes made of mixtures of 1,2-palmitoyloleoyl-sn-glycero-3-phosphocholine (POPC) and 1,2-palmitoyloleoyl-sn-glycero-3-phosphoserine (POPS) lipids and doped with the phase sensitive fluorescence probe Laurdan. ${ }^{38}$ Fluorescence and spectroscopic computational molecular dynamic simulations supported that liposomes made of 4:1 POPC: POPS (i.e. $20 \%$ content of anionic lipid) had higher adsorption levels of monovalent cations, in comparison with zwitterionic neutral vesicles. Furthermore, $\mathrm{Na}^{+}$was found to be the strongest cation adsorbed, followed by $\mathrm{K}^{+}$and then $\mathrm{Cs}^{+}$. The rigidification of the membranes was explained by the formation of lipid-cation complexes via the coordinating oxygen atoms in carbonyl groups of hydrophilic lipid head groups to the respective cations.

\subsection{Adsorption of active molecules at the water-membrane interface}

Considering the adsorption of active components to membrane surfaces is important both during the construction of photoactive liposomes and during the light-induced chemical reactions itself, electrostatic and hydrophobic interactions play a major role in the overall photocatalytic scenario. In terms of energy, the supramolecular adsorption of molecules onto lipid bilayers can be described in terms of a solvation-desolvation process, where all supramolecular forces describing the interaction between the functional molecule and the membrane have to be considered, i.e., charges and hydrophobicity indeed, but also $\pi-\pi$ stacking, dipolar interactions, van der Waals interactions, hydrogen bonding, and ligand coordination to metals. Altogether, these interactions combine into a free Gibbs energy of adsorption of the photoactive molecule to the membrane $\left(\Delta G_{\text {ads }}\right)$ that is composed of an enthalpy $\left(\Delta H_{\text {ads }}\right)$ and an entropy $\left(\Delta S_{\text {ads }}\right)$ term (eqn (14), Fig. 17).

$$
\Delta G_{\mathrm{ads}}=\Delta H_{\mathrm{ads}}-T \cdot \Delta S_{\mathrm{ads}}
$$

Both $\Delta H_{\text {ads }}$ and $\Delta S_{\text {ads }}$ have the following contributions:

(a) Supramolecular interaction between the functional molecule and lipid membrane.

(b) Solvation of the functional molecule and its counter anions/cations in the aqueous phase.

(c) Solvation of the lipid membrane and its counter anions/ cations in the aqueous phase.

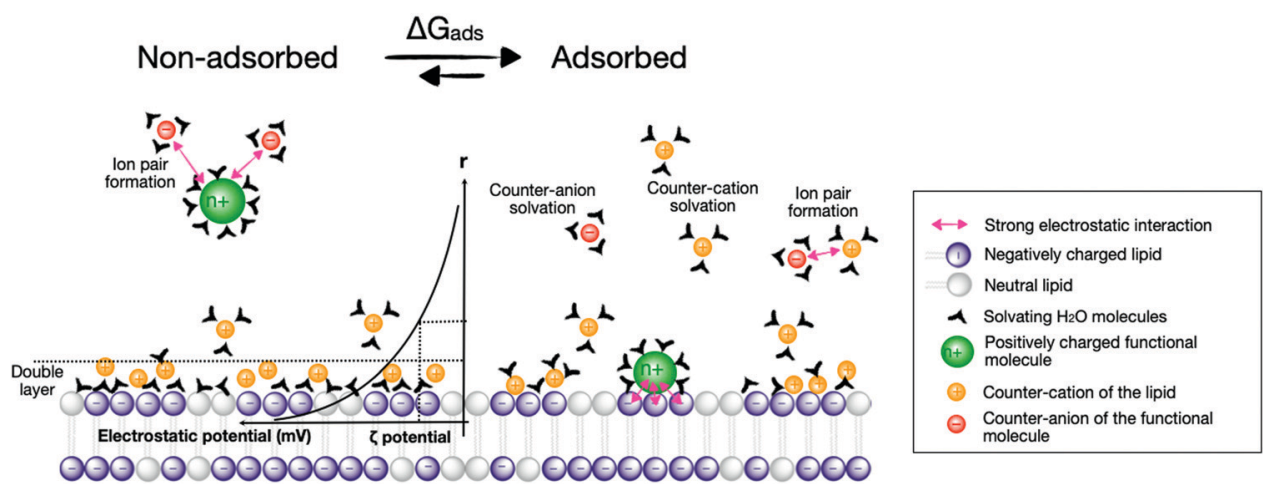

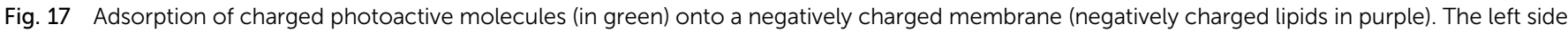

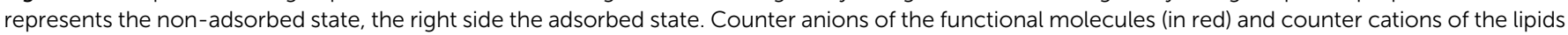

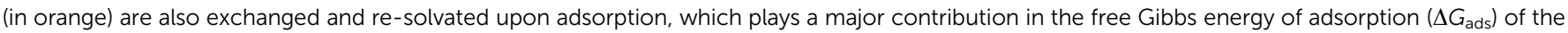

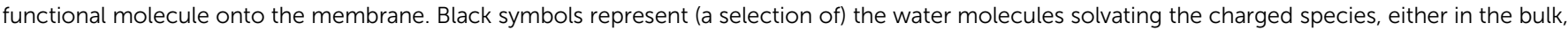

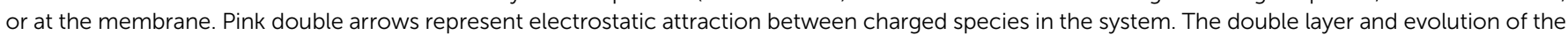
electrostatic potential and the zeta $(\zeta)$ potential are shown as a function of the distance $(r)$ from the membrane. 
(d) Ion pair formation between the counter anions and counter cations, which are released from the membrane upon adsorption.

Negatively charged lipid bilayers are normally surrounded by $\mathrm{NH}_{4}^{+}, \mathrm{Na}^{+}$or $\mathrm{K}^{+}$counter-cations, and positively charged metal complexes bring along anions such as $\mathrm{Cl}^{-}$or $\mathrm{PF}_{6}{ }^{-}$. Upon adsorption of a positively charged functional molecule to the surface of negatively charged lipid bilayers the counter-ions can be exchanged, a process that has thermodynamic consequences. In the exemplary case of the adsorption of one dicationic functional molecule such as $\left[\mathrm{Ru}(\mathrm{tpy})(\mathrm{bpy})\left(\mathrm{OH}_{2}\right)\right]^{2+}\left(\mathrm{tpy}=2,2^{\prime}: 6^{\prime}, 2^{\prime \prime}\right.$-terpyridine $)$ onto negatively charged DMPG based lipid bilayers involves the desorption of two $\mathrm{Na}^{+}$from the bilayer surface. ${ }^{51}$ Depending on the respective ion pairing and solvation energies and entropies, this process can either be endothermic or exergonic due to a significant energetic contribution from the entropy term of ion release (two equivalents of $\mathrm{Na}^{+}$cations) to the bulk. ${ }^{51}$

\subsection{Reaction dynamics at water-membrane interfaces}

Reaction dynamics differ between vesicles-supported reactions and reactions in homogeneous solutions. In a homogeneous solution, components interact in a three-dimensional manner, whereas in vesicles components are normally immobilised on a two-dimensional lipid bilayer, which forms the platform of the photoreactions. One method to study the effects of reduced dimensionality on reaction kinetics is bimolecular fluorescence quenching with PSs and quenchers attached to the membrane. For three-dimensional bimolecular reactions, fluorescence quenching with excess of quencher molecules will show a normal exponential decay of fluorescence $v s$. time, with a rate law that is pseudo-first order in quencher concentration [Q]. For reactants diffusing in $2 \mathrm{D}$ within the vesicle interface, the fluorescence decay will instead be strongly non-exponential. This can be qualitatively understood by considering the concentration profile of quenchers as a function of distance from excited molecules, $[\mathrm{Q}]=f(r)$ (Fig. 18). At the time of excitation $(t=0)$ the quenchers are randomly distributed in solution and [Q] will be independent on $r$. The excited PSs that are next to a quencher will react rapidly, and the remaining excited PSs will be the ones with larger distance to the nearest $Q$. With time, the reaction will consume all quenchers at a short distance, leaving a "concentration hole" around the remaining excited states.
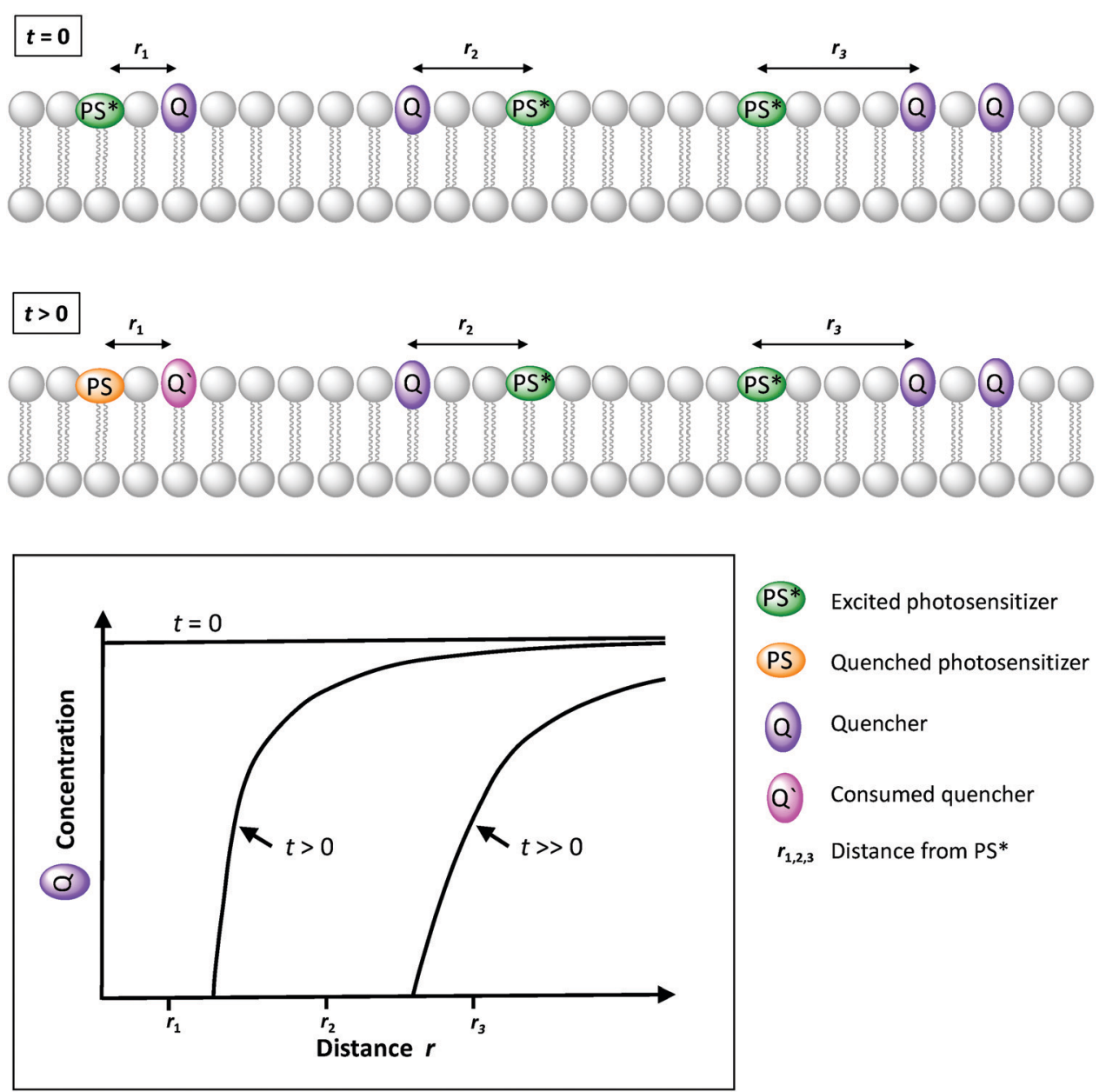

PS* Excited photosensitizer

PS Quenched photosensitizer

Q Quencher

Q. Consumed quencher

$r_{1,2,3}$ Distance from PS*

Fig. 18 Qualitative diffusion-reaction dynamics at lipid bilayer surfaces containing PS and quenchers $(Q)$ at various distances $\left(r_{1,2,3}\right)$ leads to a time-dependent concentration profile of $Q$ as a function of distance from PS*. For three-dimensional (homogeneous) solution, the concentration profile rapidly becomes constant (qualitatively similar to the profile labelled " $t>0$ "), and the usual pseudo-first order rate constant is obtained. With two-dimensional diffusion instead, the concentration profile keeps evolving and the kinetics is non-exponential. The concentration profiles shown are qualitative and not mathematically exact. 

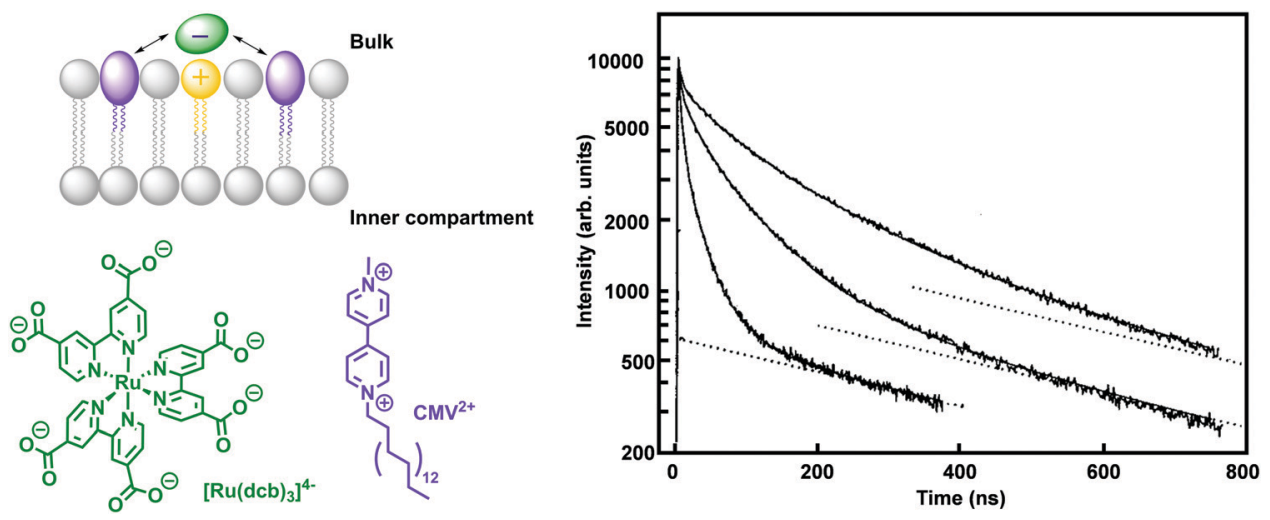

Fig. 19 Left: Example of homogeneous PS $\left[\mathrm{Ru}(\mathrm{dcb})_{3}\right]^{4-}$ (green) electrostatically associated to egg lecithin vesicles doped with cationic surfactants (yellow), and amphiphilic $\mathrm{CMV}^{2+}$ (purple) as membrane-immobilised quencher. Right: Representative time-resolved luminescence decay of $\left[\mathrm{Ru}(\mathrm{dcb})_{3}\right]^{4-}$ in the presence of $\mathrm{CMV}^{2+}$, showing strongly non-exponential behaviour (the dotted line indicate luminescence from a fraction of $\left[\mathrm{Ru}(\mathrm{dcb})_{3}\right]^{4-}$ not bound to the vesicles). Adapted with permission from L. Hammarström, T. Norrby, G. Stenhagen et al., J. Phys. Chem. B, 1997, 101, 7494-7504. Copyright (1997) American Chemical Society. ${ }^{55}$

At the same time, diffusion of quenchers from the bulk to the vesicle interface will occur to balance the concentration. In a threedimensional, homogeneous solution, reaction and diffusion rates rapidly equilibrate to set up a steady-state concentration profile of quenchers that then remains for the rest of the reaction, resulting in single-exponential kinetics. When the concentration profile still changes, however, quenching is faster than given by the long-time rate constant. This transient effect in fluorescence quenching is well established ${ }^{52}$ and is important at times after excitation $t \ll R^{2} /$ $D_{\mathrm{PSO}}$, where $R$ is the intermolecular reaction distance and $D_{\mathrm{PSO}}$ is the sum of the diffusion coefficients of PS and quencher. For small molecules in a low-viscosity solvent, this is typically at $t<100 \mathrm{ps}$.

For diffusion in lower dimensions, such as within a twodimensional lipid bilayer, diffusion can never keep up with reaction, and the "concentration hole" will keep growing as the reaction proceeds. The fluorescence decay is then much more complicated, so that numerical fitting of experimental data becomes unfeasible. An approximate solution for the time dependence of the fluorescence intensity in the case of diffusioncontrolled, two-dimensional quenching is given in eqn (15), ${ }^{53}$ where $\tau_{0}$ is the lifetime of the PS in the absence of quencher and $[\mathrm{Q}]$ is the concentration of quencher. The solution for the case of reaction-control (slower than the diffusion limit) is somewhat more complex. ${ }^{54}$ For very slow quenching, i.e. low reactivity even at contact distance, $[\mathrm{Q}]$ near the PSs will be approximately equal to the bulk value, and the quenching kinetics should again be simple and follow a single exponential.

$$
\frac{I(t)}{I(t=0)}=\exp \left\{-t / \tau_{0}-7.44[\mathrm{Q}] R\left(D_{\mathrm{PSQ}} t\right)^{\frac{1}{2}}-2.28[\mathrm{Q}] D_{\mathrm{PSQ}} t\right\}
$$

An example where eqn (15) was used to study electron transfer at the interface of vesicles is shown in Fig. 19. The ruthenium $\mathrm{PS}\left[\mathrm{Ru}(\mathrm{dcb})_{3}\right]^{4-}$ ( $\mathrm{dcb}=4,4^{\prime}$-dicarboxylate-2,2'-bipyridine $)$ was electrostatically associated to egg lecithin vesicles doped with cationic surfactants, and amphiphilic $\mathrm{CMV}^{2+}$ as quenchers. ${ }^{55}$ The good agreement with eqn (15) suggests that $\left[\mathrm{Ru}(\mathrm{dcb})_{3}\right]^{4-}$ was localised very close to the surface, with negligible diffusion orthogonal to the membrane surface. The diffusion coefficient obtained from a fit to the data was comparable to that of amphiphiles in bilayers, $(6 \pm 2) \times 10^{-11} \mathrm{~m}^{2} \mathrm{~s}^{-1}{ }^{55}$ For comparison: diffusion coefficients in homogeneous solution are on the order of $1 \times 10^{-9} \mathrm{~m}^{2} \mathrm{~s}^{-1} .{ }^{56}$ As a last remark, these kinetic effects are not only important for energy transfer; eqn (15) is also valid for electron transfer, e.g. the non-fluorescent reactions of for instance an oxidised or reduced PS, in its ground state, with membrane-bound catalysts.

\section{Conclusion and future prospects}

Although it is difficult to predict whether liposomes and membrane-based artificial photosynthetic systems will play any role in solving the energy crisis, they represent an attractive and under-explored concept that complements traditional semiconductor-based developments to drive photocatalysis. In particular, they allow interrogating the coupling of two halfreactions in a single photocatalytic system, and provide a framework for developing innovative solution for avoiding charge recombination and back reactions - the main challenge in photocatalytic artificial photosynthesis. Liposome- and lipid bilayer-based photocatalytic systems also represent a great opportunity to improve our fundamental understanding of supramolecular chemistry, not only in static terms, but also dynamically. In static terms, because one needs to understand how electrostatic forces, hydrophobicity, coordination chemistry, van der Waals interactions, and $\pi-\pi$ stacking, can be combined to organise molecules in space and achieve dissymmetric assemblies where electrons get a chance to flow faster in one direction than in the other. Photocatalytic liposomes will also improve our understanding of dynamic self-assembly, because the time-dependent nature of photochemical processes allows for probing how supramolecular chemistry evolves in time under the action of light absorption, energy transfer, and electron transfer, while covalent chemistry often 
offers decomposition as the final outcome of photoelectron transfer. Photocatalytic lipid bilayers also pave the way towards new strategies to regenerate photochemically or mechanically damaged components, possibly by self-healing processes. These are aspects that are very difficult to implement in covalent systems, but also in most solid-state-materials.

Photochemistry on lipid bilayers, however, also represent a formidable challenge. First, photochemically active liposomes are nowadays beyond the limit of what can be modelled by computational methods. It is hence usually difficult to build an accurate theoretical model that fits experimental data. Second, they make time-resolved spectroscopy methods particularly challenging because of the high light scattering generated by liposomes, the size of which precisely fits with the wavelength of visible light. Hopefully, these challenges will be overcome by combining experimental work on photocatalytic liposomes with the knowledge generated by alternative self-assembled electro- or photo-catalytic systems. For example, hybrid photocatalytic electrodes combine small artificial molecules as electron relays and enzymes as catalysts, to transform light energy into current. Photocatalytic soap films have also been proposed, for example in the SOFIA project (GAN 828838), to convert sun energy into a chemical fuel. Vesicle-based compartmentalisation catalysis will also be very useful, as it aims at combining several molecular or enzyme-based catalyst using a vesicle-in-a-vesicle strategy. In such assemblies, small molecules do move across membranes to perform a series of chemical reactions, while the catalysts stay isolated from each other by the different membranes of the compartmentalised system. Ultimately, we are convinced that full understanding of a process as complicated as natural photosynthesis, requires scientists to be able of making, with their own hands, an artificial (or semi-artificial) photocatalytic system that can perform the same function as the natural one: transforming the fleeting, transient energy of photons in a sunlight beam, into the stable chemical energy of a chemical bond.

\section{Conflicts of interest}

There are no conflicts to declare.

\section{Acknowledgements}

This project has received funding from the European Union's Horizon 2020 research and innovation program FETOPEN 2018-2020 under grant agreement \# 828838 - SoFiA. A. Pannwitz gratefully acknowledges the Vector Foundation. D. M. Klein thanks the Netherlands Organization for Scientific Research (NWO) for an HRSMC PhD fellowship. C. Casadevall acknowledges the European Commission for an Horizon 2020 Marie SkłodowskaCurie Individual Fellowship (890745-SmArtC). C. Casadevall and E. Reisner acknowledge the Biotechnology and Biological Sciences Research Council (BBSRC) for financial support (BB/ S00159X/1).

\section{References}

1 W. Lubitz, H. Ogata, O. Rüdiger and E. Reijerse, Chem. Rev., 2014, 114, 4081-4148.

2 X. Fang, S. Kalathil and E. Reisner, Chem. Soc. Rev., 2020, 49, 4926-4952.

3 I. Rojdestvenski, A. G. Ivanov, M. G. Cottam, A. Borodich, N. P. A. Huner and G. Oquist, Biophys. J., 2002, 82, 1719-1730.

4 B. Daum, D. Nicastro, J. Austin, J. R. McIntosh and W. Kühlbrandt, Plant Cell, 2010, 22, 1299-1312.

5 A. Stikane, E. T. Hwang, E. V. Ainsworth, S. E. H. Piper, K. Critchley, J. N. Butt, E. Reisner and L. J. C. Jeuken, Faraday Discuss., 2019, 215, 26-38.

6 M. Hansen, S. Troppmann and B. König, Chem. - Eur. J., 2016, 22, 58-72.

7 J. N. Robinson and D. J. Cole-Hamilton, Chem. Soc. Rev., 1991, 20, 49-94.

8 J. Flores, B. M. White, R. J. Brea, J. M. Baskin and N. K. Devaraj, Chem. Soc. Rev., 2020, 49, 4602-4614.

9 F. A. Armstrong and J. Hirst, Proc. Natl. Acad. Sci. U. S. A., 2011, 108, 14049-14054.

10 E. Rideau, R. Dimova, P. Schwille, F. R. Wurm and K. Landfester, Chem. Soc. Rev., 2018, 47, 8572-8610.

11 H.-J. Choi and C. D. Montemagno, Nano Lett., 2005, 5, 2538-2542.

12 M. C. M. van Oers, F. Rutjes and J. C. M. van Hest, Curr. Opin. Biotechnol, 2014, 28, 10-16.

13 D. Hvasanov, J. R. Peterson and P. Thordarson, Chem. Sci., 2013, 4, 3833-3838.

14 T. F. Tadros, An Introduction to Surfactants, De Gruyter, 2014.

15 D. Marsh, Handbook of Lipid Bilayers, CRC Press, 2013.

16 A. Leo, C. Hansch and D. Elkins, Chem. Rev., 1971, 71, 525-616.

17 J. N. Israelachvili, Intermolecular and Surface Forces, Academic Press, 2011.

18 D. M. Arias-Rotondo and J. K. McCusker, Chem. Soc. Rev., 2016, 45, 5803-5820.

19 S. Troppmann and B. König, Chem. - Eur. J., 2014, 20, 14570-14574.

20 G. D. Scholes, G. R. Fleming, A. Olaya-Castro and R. van Grondelle, Nat. Chem., 2011, 3, 763-774.

21 C. A. Puckett and J. K. Barton, J. Am. Chem. Soc., 2007, 129, 46-47.

22 M. Hansen, F. Li, L. Sun and B. König, Chem. Sci., 2014, 5, 2683-2687.

23 B. Limburg, J. Wermink, S. S. Van Nielen, R. Kortlever, M. T. M. Koper, E. Bouwman and S. Bonnet, ACS Catal., 2016, 6, 5968-5977.

24 N. Ikuta, S. Y. Takizawa and S. Murata, Photochem. Photobiol. Sci., 2014, 13, 691-702.

25 B. Limburg, E. Bouwman and S. Bonnet, J. Phys. Chem. B, 2016, 120, 6969-6975.

26 B. Limburg, E. Bouwman and S. Bonnet, Chem. Commun., 2015, 51, 17128-17131.

27 N. Sugiyama, M. Toyoda and Y. Amao, Colloids Surf., A, 2006, 284-285, 384-387.

28 Y. Amao and I. Okura, J. Mol. Catal. A: Chem., 1996, 105, 125-130. 
29 F. Franco, S. Fernández and J. Lloret-Fillol, Curr. Opin. Electrochem., 2019, 15, 109-117.

30 P. Garrido-Barros, C. Gimbert-Suriñach, R. Matheu, X. Sala and A. Llobet, Chem. Soc. Rev., 2017, 46, 6088-6098.

31 V. Radu, S. Frielingsdorf, S. D. Evans, O. Lenz and L. J. C. Jeuken, J. Am. Chem. Soc., 2014, 136, 8512-8515.

32 Y. Pellegrin and F. Odobel, C. R. Chim, 2017, 20, 283-295.

33 G. Steinberg-Yfrach, P. A. Liddell, S.-C. Hung, A. L. Moore, D. Gust and T. A. Moore, Nature, 1997, 385, 239-241.

34 M. Andersson, L. Hammarström and K. Edwards, J. Phys. Chem., 1995, 99, 14531-14538.

35 G. Steinberg-Yfrach, J.-L. Rigaud, E. N. Durantini, A. L. Moore, D. Gust and T. A. Moore, Nature, 1998, 392, 479-482.

36 I. O. L. Bacellar, M. C. Oliveira, L. S. Dantas, E. B. Costa, H. C. Junqueira, W. K. Martins, A. M. Durantini, G. Cosa, P. Di Mascio, M. Wainwright, R. Miotto, R. M. Cordeiro, S. Miyamoto and M. S. Baptista, J. Am. Chem. Soc., 2018, 140, 9606-9615.

37 S. Troppmann, E. Brandes, H. Motschmann, F. Li, M. Wang, L. Sun and B. König, Eur. J. Inorg. Chem., 2016, 554-560.

38 P. Jurkiewicz, L. Cwiklik, A. Vojtíšková, P. Jungwirth and M. Hof, Biochim. Biophys. Acta, Biomembr., 2012, 1818, 609-616.

39 A. Pannwitz, H. Saaring, N. Beztsinna, X. Li, M. A. Siegler and S. Bonnet, Chem. - Eur. J., 2021, 27, 3013-3018.

40 J. Sabın, G. Prieto, J. M. Ruso, R. Hidalgo-Álvarez and F. Sarmiento, Eur. Phys. J. E: Soft Matter Biol. Phys., 2006, 20, 401-408.

41 S. Paula, A. G. Volkov, A. N. Van Hoek, T. H. Haines and D. W. Deamer, Biophys. J., 1996, 70, 339-348.

42 B. Maherani, E. Arab-Tehrany, A. Kheirolomoom, D. Geny and M. Linder, Biochimie, 2013, 95, 2018-2033.
43 L. Hammarström, M. Almgren and T. Norrby, J. Phys. Chem., 1992, 96, 5017-5024.

44 L. M. Hays, J. H. Crowe, W. Wolkers and S. Rudenko, Cryobiology, 2001, 42, 88-102.

45 I. D. Johnson, Molecular Probes Handbook: A Guide to Fluorescent Probes and Labeling Technologies, Life Technologies Corporation, 2010.

46 C. C. Page, C. C. Moser, X. Chen and P. L. Dutton, Nature, 1999, 402, 47-52.

47 E. Altamura, F. Milano, R. R. Tangorra, M. Trotta, O. H. Omar, P. Stano and F. Mavelli, Proc. Natl. Acad. Sci. U. S. A., 2017, 114, 3837-3842.

48 S. Bhosale, A. L. Sisson, P. Talukdar, A. Fürstanberg, N. Banetji, E. Vauthey, G. Bollot, J. Mareda, C. Röger, F. Würthner, N. Sakai and S. Matile, Science, 2006, 313, 84-86.

49 A. Perez-Velasco, V. Gorteau and S. Matile, Angew. Chem., Int. Ed., 2008, 47, 921-923.

50 B. Limburg, E. Bouwman and S. Bonnet, ACS Catal., 2016, 6, 5273-5284.

51 A. Bahreman, M. Rabe, A. Kros, G. Bruylants and S. Bonnet, Chem. - Eur. J., 2014, 20, 7429-7438.

52 T. L. Nemzek and W. R. Ware, J. Chem. Phys., 1975, 62, 477-489.

53 C. S. Owen, J. Chem. Phys., 1975, 62, 3204-3207.

54 B. Medhage and M. Almgren, J. Fluoresc., 1992, 2, 7-21.

55 L. Hammarström, T. Norrby, G. Stenhagen, J. Mårtensson, B. Åkermark and M. Almgren, J. Phys. Chem. B, 1997, 101, 7494-7504.

56 J. Moldenhauer, M. Meier and D. W. Paul, J. Electrochem. Soc., 2016, 163, H672-H678. 